\title{
Complementarities, Coordination, and Culture
}

\author{
Christopher Ellis \\ Jon C. Thompson \\ Jiabin $\mathrm{Wu}$
}

CESIFO WORKING PAPER NO. 5949

CATEgory 1: Public FinANCE

JUNE 2016

An electronic version of the paper may be downloaded

- from the SSRN website: Www.SSRN.com

- from the RePEc website: $\quad$ www.RePEc.org

- from the CESifo website: www.CESifo-group.org/wp 


\title{
Complementarities, Coordination, and Culture
}

\begin{abstract}
We develop a new model of the intergenerational transmission of culture based on the labor market characteristics of different cultural types. Following Borjas $(1994,1995)$ we assume that cultural heterogeneity increases labor productivity due to skill complementarities, however following Jackson and Xing (2014) we also assume that cultural heterogeneity hampers communication. We model this as a game in which individuals first engage in uniformly random matching, and then once matched play a coordination game. Other-type matches are thus potentially more productive, but also less likely to coordinate. We show that this set up can replicate many of the seminal results of Bisin and Verdier (2001) without assuming imperfect empathy. This means that we do not face the inherent difficulties that such models involve when making welfare statements. Further, we are able to obtain new results concerning the efficient size of a cultural minority, the effects of tax and welfare programs on the size and welfare of minorities, and the relationship between the intensity of social interaction and size and welfare of a minority. In an extension of the model we introduce "intermediaries" who may either facilitate other-type matching or improve after-match coordination. We then ask which parent population, the minority or majority, the intermediaries arise from and explore their implications for the equilibrium and welfare.
\end{abstract}

JEL-Codes: A140, C730, D100, Z130.

Keywords: cultural transmission, coordination games, social interaction, minorities, intermediaries, tax and welfare programs.

\author{
Christopher Ellis \\ Department of Economics \\ University of Oregon \\ USA - 97403-1285 Eugene OR \\ cjellis@uoregon.edu
}

Jon C. Thompson

Atkinson Graduate School of Management

Willamette University

USA - Salem, Oregon 97301

jcthomps@willamette.edu
Jiabin $W u$

Department of Economics

University of Oregon

USA - 97403-1285 Eugene OR

jwu5@uoregon.edu

June 5, 2016

We thank Bruce McGough for his helpful comments. 


\section{Introduction}

In November 2015 UNHCR the United Nations Refugee Agency reported that in the preceding four years, in response to conflict in their homeland, over 500,000 Syrian citizens had sought asylum in Europe, with the preponderance of these people seeking to enter Germany. Generally public attitudes in the largest European Union countries are unsympathetic towards immigration. In response to the question, "Is immigration a problem not an opportunity" more than $40 \%$ of the respondents from the populations of the UK, France, Italy, Portugal, the Netherlands and Spain responded "yes". Survey participants, particularly those in the UK, voiced concerns over "social cohesion" and competition for jobs.17 Yet studies for the UK indicate that immigration actually has a small positive effect on average wages, and that immigrants are net fiscal contributors, paying more in taxes than the value of services and transfers they receive $2^{2}$ The plight of the Syrian refugees highlights a number of important and interesting questions concerning the benefits and costs of the presence of a cultural minority in a population. It further raises the question of whether these costs and benefits may be expected to persist, that is whether the immigrant minority may be expected to assimilate into their host populations or remain culturally distinct. These are questions of cultural transmission. There is an extensive literature on this topic, the modern version of which was pioneered by Bisin and Verdier (1998) and is surveyed in Bisin and Verdier (2010). The key idea in this literature is imperfect empathy whereby a parent uses their own preferences to evaluate the utility of their offspring, not the offsprings own preferences as in the standard results on altruism. Imperfect empathy provides an incentive for a parent to incur a cost so as to socialize a child to share their own cultural traits. If it is then assumed that the alternative to parental socialization is for the offspring to be socialized by a randomly chosen role-model from the general population, then the analysis can explain the persistence of cultural minorities within a population $3^{3}$ This approach, while appealing

\footnotetext{
${ }^{1}$ Source "The Migratory Observatory at the University of Oxford" http://www.migrationobservatory.ox.ac.uk/briefings/uk-public-opinion-toward-immigration-overallattitudes-and-level-concern

${ }^{2}$ See Dustmann, Frattini and Preston (2005), (2013) on labor market effects, and Dustmann and Frattini (2014) for results on fiscal contributions

${ }^{3}$ As the proportion of the population that are not of the minority approaches $100 \%$ a minority child will almost certainly become a member of the majority if socialized by a random stranger, hence a cultural mi-
} 
in many ways, explains cultural choices by assuming a preference over preferences together with an assumed specific technology for socialization $4^{45}$ In what follows we develop a simple model of cultural choices based on different primitives. Our model will be based on the nature of random matching and the productivity characteristics of different cultural types. Our approach is inspired by early observations made by Borjas (2003) and Lazear (1999a,b), first that immigrants may bring skills to the labor market complementary to those posesed by natives, and, second, they may introduce costs via difficulties in communication. We base our model of cultural choice on these observations in a very natural way. We assume that agents in an economy consist of one of two types, these types are culturally distinct and poses complementary skills $\sqrt{6}^{7}$ The agents in the economy are subject to uniformly random matching, and once matched play a coordination game 8 If the players coordinate on an equilibrium then a surplus is realized and allocated between the participants via Nash

nority parent who is culturally intolerant will choose to socialize the child themselves, ensuring the continued existence of the minority.

${ }^{4}$ This is not to say that several different cultural transmission mechanisms cannot be found in the literature, Bisin and Verdier's (2010) analysis of homogamous marriages is particularly interesting, but to obtain the long run persistence of a cultural type requires in each case that direct socialization by parent(s) becomes more attractive as the proportion of the minority in the population falls.

${ }^{5}$ In a recent paper Giusta, Hashimzade and Myles (2016) argue that parents from an immigrant population must trade off the labor market advantages of schooling and the cultural costs arising from the schools socializing their children in the host culture. Like Bisin and Verdier they essentially assume that the parents have a preference over preferences, however their socialization dynamic, working through schooling choice, yields a richer and more intuitively appealing set of results. Here culture can actually evolve over time.

${ }^{6}$ Recent evidence on the effects of ethnic diversity in teams has been supplied by Hoogendoorn and van Praag (2012), who study 45 real student companies that differ primarily in their ethnic composition. They find that if there is sufficient diversity then this is productivity enhancing. Ottaviano and Peri $(2005,2006)$ find that cultural diversity, as measured by the number of languages spoken, is productivity increasing at the US city level. Hong and Page $(2001,2004)$ explain the greater productivity of diverse group by their greater ability to problem solve as a consequence of employing different heuristics. Also see Page (2007) for a book length discussion. Niebuhr (2010) studies the production of patents by region in Germany, and shows that cultural diversity enhances the performance of regional R\&D sectors. Sparber (2009) studies aggregate productivity in US industries for the 1980-2000 period, and finds that those sectors that employ many workers in creative decision making or customer services roles experience productivity gains from increased racial diversity, however industries characterized by high levels of group effort experience decreasing productivity consistent with coordination and communication problems. Ahern et al (2015) argues that cultural diversity could increase the likelihood of a successful merger because employees with different cultural values could introduce alternatives to the status-quo which lead to greater efficiency. However, cultural diversity may reduce the post-merger coordination between the employees of each firm due to impediments such as mistrust and misunderstanding.

${ }^{7} \mathrm{~A}$ complimentary literature looks at gender diversity and productivity in the workplace, a recent study by Ellison and Mullin (2014) finds that diversity is negatively related to the provision of social goods, trust, cooperation etc., and positively related to productivity.

${ }^{8}$ For a survey of matching functions see Petrongolo and Pissarides (2001). 
bargaining. An agent's cultural type effects their expected payoffs and ultimately the equilibrium distribution of types in three ways; first the types have complementary skills such that cross cultural "other-type" matches are potentially more productive than "own-type" matches, second the probability of equilibrium coordination is lower for other-type matches versus own-type matches reflecting their cultural dissimilarities, thirdly and finally the types possess different threat points that effect the distribution of surplus following successful coordination. An equilibrium is characterized by the proportion of the types in the population, which for either type may be zero, one, or some intermediate value indicating the persistence of a minority $9^{9}$ In the context of our motivating example above this allows us to predict what might happen to Syrian refugees that take up residency in Germany; if they will integrate or remain culturally distinct, and if they do integrate how long will it take and why? We may also explore if the integration of these immigrants is a good thing from a social welfare perspective, and if the immigration and diversity policies that have been practiced around Europe are likely to promote the integration of new migrants. We shall be able to comment on each of these topical issues.

In the next two sections we develop a basic static model, derive the distribution of types that would be chosen by a benevolent planner and compare this to a market solution where the agents choose their own type so as to maximize expected payoffs. We show that for an interior equilibrium the market solution involves too few agents choosing the minority culture ${ }^{10} 11$ In section 4 we explore the effects of tax and welfare systems on the equilibrium proportions of the cultural types. We show that lump-sum taxes/transfers between types are sufficient instruments to implement the utilitarian social welfare optimum. Further if these taxes are set via democratic voting, and hence maximize the expected payoff to a member

\footnotetext{
${ }^{9}$ That a good theory of cultural transmission must admit equilibria characterized both by full integration of a minority, and by the minority's persistence, is highlighted nicely by counterposing the examples given by Bisin and Verdier (2010) and Giusta, Hashimzade and Myles (2016). The former motivate their analysis by pointing to the continued existence and cultural distinctiveness of the Orthodox Jewish community in the US. The latter motivate their analysis by pointing to the complete assimilation of the Jewish community in the East End of London in the late nineteenth and early twentieth centuries.

${ }^{10}$ The static nature of our preliminary analysis may seem too restrictive, but in later sections we introduce two types of dynamics and demonstrate that the key results of the static model are not reliant on the static assumption.

${ }^{11}$ Allowing agents to choose their own type might seem a strange assumption, but as we show in the dynamic sections that follow this is just short hand for a steady state equilibrium where altruistic parents probabilistically choose their children's cultural type.
} 
of the majority cultural type, then the mix of cultural types in equilibrium is the same as at the social welfare optimum. However, the simplest possible welfare scheme, whereby all incomes are taxed at a constant rate with the revenues used to supply a public service does not effect the market equilibrium proportions of the two cultural types.

In section 5 we explore the implications of the intensity of social interaction for the equilibrium proportions of the two cultural types, and for the welfare properties of the equilibrium. We model greater social interaction by allowing for multiple rounds of matching per period, where more rounds represents greater social interaction. We show that a greater intensity of social interaction pushes the market equilibrium towards the welfare optimum, and is Pareto improving.

In section 6 we introduce dynamics into the framework. We assume that parents are fully altruistic. We explore two socialization dynamics; one based on increasing convex costs of socialization, and a second based on Bisin and Verdier's (2001) model but without employing imperfect empathy. In the case where parents costs of changing their children's culture are increasing and convex, we find that the interior equilibrium may or may not be stable. Furthermore, even when the interior steady state equilibrium is stable, the speed of convergence of the equilibrium may be faster or slower than is welfare optimal. We explain the distortions behind this finding, and how it relates to the "cultural environment" defined by the within group cohesion of the cultural groups, and the "cultural distance" between them. This allows us to gain some insights into why some governments may adopt policies to change the rate of assimilation of a minority. In the case where a parent pays to increase the probability that their child will be of their own cultural type as in Bisin and Verdier (2001), we find that the interior equilibrium is always asymptotically stable.

In section 7 we make the obvious observation that there are rents to be earned if the matching process can be improved, or if rates of coordination within matches can be increased. We posit the emergence of "intermediaries" that perform these functions. In the context of improving matching we suppose that intermediaries perform the service of providing cross-cultural introductions. We show that since intermediaries do not fully internalize the benefits of the improvements in matching they facilitate then there will be fewer of them than is socially efficient. When intermediaries improve coordination there are several possi- 
bilities depending on which characteristics are important for coordination. If coordination by intermediaries depends on "social traits" such as language proficiency or knowledge of social customs, then we argue that coordination is limited to the rate of the cultural type that is poorest at coordinating in an own-type (same culture) match. The idea being an intermediary cannot understand a type better than they understand themselves. Here the intermediaries are shown to always emerge from the majority type. Alternatively, if coordination by an intermediary depends on "personal traits" such as empathy or diplomatic skill, then intermediaries may be drawn form either group dependent on their (culturally determined) endowment of these traits. Finally, if the ability of intermediaries to improve coordination depends on their cultural experiences, intermediaries are shown to emerge from the minority type because minority type agents have a high probability of encountering majority type agents and thus they have greater experience in dealing with cross-cultural matches.

In section 8 we provide a conclusion and some suggestions for further study.

\section{Benchmark Model}

\section{$2.1 \quad$ Structure}

The economy is populated by $n$ agents, which we normalize to a population of 1 . Agents may be of one of two cultural types $i$ or $j$. The proportion of agents of cultural type $i$ in the population is denoted $\lambda$ so the proportion $1-\lambda$ are of type $j$, where $\lambda \in[0,1]$. Production and hence the payoffs in this economy result from pairs of agents randomly matched to play a coordination game. Each may choose an action $x_{k} \in\{a, b\}, k \in\{i, j\}$. Successful coordination involves each player in the matched pair choosing the same action 12

\footnotetext{
${ }^{12}$ Our work does share some commonalities with Berliant and Fujita (2008) who model the creation of knowledge. In their work having more ideas in common improves communication between pairs of potential knowledge creators.
} 


\subsubsection{Rents}

The total rents generated by a match for given action choices are written $R\left(x_{k}, x_{h}\right)$. We suppose

Assumption 1. (a) $R\left(a_{k}, a_{h}\right)=R\left(b_{k}, b_{h}\right)>R\left(a_{k}, b_{h}\right)=R\left(b_{k}, a_{h}\right)=0, k, h \in\{i, j\}$, (b) $R\left(a_{k}, a_{h}\right)=R\left(b_{k}, b_{h}\right)>\min _{k}\left\{R\left(a_{k}, a_{k}\right)\right\} k, h \in\{i, j\}, k \neq h$.

Part (a) tells us that a failure to coordinate yields zero rents and coordination yields positive rents.13 Part (b) tells us that other-type coordination yields higher rents than own-type coordination for at least one type 14 The idea here is that each type brings a cultural-skill to a match, and that for other-type matches these skills are complementary and yield higher rents than in own-type matches.

\subsubsection{Payoffs}

We write the players payoffs as $P_{k, h}\left(x_{k}, x_{h}\right), k, h \in\{i, j\}$. Where the first index of $P$ indicated the type of the player receiving the payoff and the second index the type of the player is matched with. In this section, we shall assume that the payoffs represent an even division of the rents for each possible match ${ }^{15}$ We have

$$
\begin{gathered}
R\left(x_{k}, x_{h}\right)=P_{k, h}\left(x_{k}, x_{h}\right)+P_{h, k}\left(x_{k}, x_{h}\right), k, h \in\{i, j\} \\
P_{k, h}\left(x_{k}, x_{h}\right)=P_{h, k}\left(x_{k}, x_{h}\right)=\left(\frac{1}{2}\right) R\left(x_{k}, x_{h}\right), k, h \in\{i, j\} .
\end{gathered}
$$

\footnotetext{
${ }^{13}$ Symmetry in rents implies that only coordination matters, not what the players coordinate on.

${ }^{14}$ We may think of this as the different types bringing different intermediate inputs into a Dixit-Stiglitz type production technology as in Alesina, Spolaore and Wacziarg (2000).

${ }^{15}$ Or there is bargaining and the players all share a common threat point. In the subsequent sections we allow division of rents to be determined by Nash bargaining between each matched pair.
} 
Further Assumption 1 and expression (2.1) jointly imply

$$
\begin{aligned}
& P_{k, h}(a, a)=P_{k, h}(b, b)>P_{k, h}(a, b)=P_{k, h}(b, a)=0, k, h \in\{i, j\} ; \\
& P_{k, h}(a, a)=P_{k, h}(b, b)>\min _{k}\left\{P_{k, k}(a, a)\right\}, k, h \in\{i, j\}, k \neq h ; \\
& P_{k, k}(a, a)=P_{k, k}(b, b) \lesseqgtr P_{h, h}(a, a)=P_{h, h}(b, b)>0, k, h \in\{i, j\}, k \neq h .
\end{aligned}
$$

Equation (2.2) tells us that a failure to coordinate yields zero payoffs while coordination yields positive payoffs,. Furthermore, coordination with an other-type yields higher payoffs than coordination with an own-type for at least one type. In this section, but only here, we strengthen this assumption such that $P_{k, h}(a, a)=P_{k, h}(b, b)>\max _{k}\left\{P_{k, k}(a, a)\right\} k, h \in$ $\{i, j\}, k \neq h$.

\subsubsection{Coordination Probabilities and Expected Payoffs}

The players are not necessarily successful in their attempts to coordinate. We write the probability that any pair successfully coordinate as $\rho_{k, h}, k, h \in\{i, j\}$. We assume

Assumption 2. (a) $\rho_{k, k}>\rho_{k, h}=\rho_{h, k}, k, h \in\{i, j\}, k \neq h$, and (b) $\rho_{i, i} \lesseqgtr \rho_{j, j}$.

The coordination probabilities capture our basic idea that is easier to coordinate with a cultural own-type, than with a cultural other-type. Here this is taken as an exogenous characteristic of the cultural type ${ }^{16}{ }^{17}{ }^{18}$

\footnotetext{
${ }^{16} \mathrm{~A}$ very nice microfoundation for the assumption that coordination within a cultural type is easier than across cultural types is supplied by Kets and Sandroni (2015). They formalize the Theory of Mind from psychology to propose the concept of an "introspective equilibrium". The idea is that each individual playing a coordination game has an initial, payoff-irrelevant, impulse towards a particular action. These impulses are correlated across individuals with within type correlations being stronger than across type correlations. In an attempting to predict the play of the player they are matched with each player inspects their own impulse and observes the type of the player they are matched with, they are aware that the other player is doing the same and that impulses are correlated. The limit of the subsequent induction process produces their best replies. This equilibrium is shown to be unique but not necessarily efficient, particularly when common cultural types are matched and have highly correlated impulses they are quite likely to pick an inefficient equilibrium. Whereas different types that are less "influenced" by their impulses and may be more likely to coordinate on the efficient equilibrium. Alternatively coordination might require communication in the spirit of Dewatripont and Tirole (2005) and communication requires more costly effort across rather than within cultures, and indeed might involve greater strategic subsitutability.

${ }^{17}$ In a recent experimental work, Jackson and Xing (2014) study the behavior of Indian and American subjects playing a coordination game. They find that the two populations tend to coordinate on different equilibria, and that they successfully coordinated significantly more frequently when match with partners from their own population as compared to when they are matched across populations.

${ }^{18}$ In the psychology literature the ability of individuals to better encode and decode affective signals that
} 
These coordination probabilities may be given some quite intuitive interpretations. $\rho_{k, k}$ may be interpreted as the internal cohesion of the $k$-type group, and $\rho_{k, h} k \neq h$ as the cultural distance between the two types. These interpretations play an important role in what follows.

The expected payoffs to each type for each possible match are simply the payoff multiplied by the probability of successful coordination 19 Hereafter, so as to economize on notation and whenever the interpretation is clear, we write $\rho_{k, h} R\left(x_{k}, x_{h}\right) \equiv \mathscr{E}_{k, h}$ for $k, h \in\{i, j\}$, and use $\mathscr{P}_{k, h}$ to denote a $k$-type player's expected payoff when matched with a $h$-type for $k, h \in\{i, j\}$. Note that in the case of even division of the rents, we have $\mathscr{P}_{k, h} \equiv \rho_{k, h} P\left(x_{k}, x_{h}\right)$ for $k, h \in\{i, j\}$. To ensure that the types potentially have an incentive to interact we make the following assumption.

Assumption 3. Other-type matches create positive expected surplus; that is $2 \mathscr{E}_{i, j}>\mathscr{E}_{i, i}+$ $\mathscr{E}_{j, j} \cdot 20$

In later sections we shall allow the types to bargain over the division of the surplus from cross cultural matches. This assumption ensures that such behavior can take place. Now given that the proportions of types $i$ and $j$ in the population are $\lambda$ and $1-\lambda$ respectively

may be verbal (but sounds rather than words) or visual (facial expressions) is referred to as the In-Group Advantage. Seminal work by Elfenbein and Ambady (2002) argues that a common culture increases the rate at which such signals are accurately transmitted. Recent persuasive evidence supporting the In-Group advantage has been provided by Sauter, Eisner, Ekman and Scott (2010).

${ }^{19}$ The implied potential trade-off between greater productivity in other-type matches, and a greater probability of coordination in own-type matches is similar to the ideas of Lazear (1999 a, b) where different skills in a production unit may increase overall productivity but may involve costs that arise due to difficult communication between people with different languages, culture and etc. In an empirical paper, O'Reilly, Williams and Barsade (1997) analyze 32 project teams and find that more diversity lead to more conflict and less communication, but controlling for the latter it also leads to higher productivity.

${ }^{20}$ Hamilton, Nickerson and Owan (2003), in a celebrated study of the garment industry, show that despite communication difficulties, teams that are more diverse in terms of skills are more productive. In a similar vein, Ottaviano and Peri (2006) find "a very robust correlation: US-born citizens living in metropolitan areas where the share of foreign-born increased between 1970 and 1990, experienced a significant increase in their wage and in the rental price of their housing. Such finding is economically significant and survives omitted variable bias and endogeneity bias". Further, Trax, Brunow and Suedekum (2015) demonstrate that for a sample of German firms an increase in cultural diversity amongst the workforce increases plant-level productivity. Stahl et al (2009) provide a meta-analysis of 108 empirical studies that concludes that in teams cultural diversity leads to productivity losses due to task conflict and decreased social integration, but productivity gains due to increased creativity and workplace satisfaction. The net effects depended on the context. 
we may write the expected payoff of a player of each type as

$$
\begin{aligned}
& E[P(i \mid \lambda)]=\lambda \mathscr{P}_{i, i}+(1-\lambda) \mathscr{P}_{i, j} \\
& E[P(j \mid \lambda)]=\lambda \mathscr{P}_{j, i}+(1-\lambda) \mathscr{P}_{j, j}
\end{aligned}
$$

We may now address the issue of cultural selection.

\subsubsection{Cultural Selection}

We initially assume that at the beginning of the game each player may select their cultural type $i$ or $j$. This has the merit of allowing us to develop our initial analysis in a simple static framework. Obviously we do not intend this to be taken literally, rather we have in mind the story that these choices are made by perfectly altruistic parents who maximize their children's utility by choosing their cultural type. In a later section we recast the analysis as a dynamic game with overlapping generations and find that the insights we gain in the static analysis maintain. Clearly the agents' choice of cultural types can lead to one of three

possibilities $\lambda=0, \lambda=1$, or $\lambda \in(0,1)$, the details of which are described in the following two propositions.

Proposition 1. In equilibrium cultural selection involves

(i) $\lambda=0$ iff $E[P(j \mid \lambda=0)]>E[P(i \mid \lambda=0)] \Leftrightarrow \mathscr{P}_{j, j}>\mathscr{P}_{i, j}$;

(ii) $\lambda=1$ iff $E[P(i \mid \lambda=1)]>E[P(j \mid \lambda=1)] \Leftrightarrow \mathscr{P}_{i, i}>\mathscr{P}_{j, i}$;

(iii) $\lambda \in(0,1)$ iff $E[P(i \mid \lambda \in(0,1))]=E[P(j \mid \lambda \in(0,1)]$

$\Leftrightarrow \lambda \mathscr{P}_{i, i}+(1-\lambda) \mathscr{P}_{i, j}=\lambda \mathscr{P}_{j, i}+(1-\lambda) \mathscr{P}_{j, j}$.

This simply tells us that for both cultural types to be chosen by some agents then the expected value of an other-type match cannot be less than an own-type match. The last part of Proposition 1 is a cultural arbitrage condition that allows us to state

Proposition 2. The interior equilibrium

(i) Is defined by $\lambda=\frac{\mathscr{E}_{i, j}-\mathscr{E}_{j, j}}{2 \mathscr{E}_{i, j}-\mathscr{E}_{i, i}-\mathscr{E}_{j, j}} \in(0,1)$;

(ii) Is unique and stable if $\mathscr{E}_{i, i}<\mathscr{E}_{i, j}$ and $\mathscr{E}_{j, j}<\mathscr{E}_{i, j}$;

(iii) Has the property $\lambda \lesseqgtr \frac{1}{2}$ as $\mathscr{E}_{i, i} \lesseqgtr \mathscr{E}_{j, j}$. 
Hence the interior equilibrium is unique and stable if other-type matches yield higher expected payoffs than own-type matches ${ }^{21}$ Further, it involves type $i$ agents being in the majority if their own-type matches yield higher expected payoffs than those of $j$-type agents. ${ }^{22}$

\subsection{Welfare Optima}

We adopt a utilitarian social welfare function with equal weight on both types such that expected social welfare is written

$$
\begin{aligned}
E[W \mid \lambda] & =\lambda E[P(i \mid \lambda)]+(1-\lambda) E[P(j \mid \lambda)] \\
& =\lambda\left[\lambda \mathscr{P}_{i, i}+(1-\lambda) \mathscr{P}_{i, j}\right]+(1-\lambda)\left[\lambda \mathscr{P}_{j, i}+(1-\lambda) \mathscr{P}_{j, j}\right]
\end{aligned}
$$

Differentiating wrt $\lambda$ and solving the resulting FOC reveals the solution

$$
\lambda^{*}=\underset{\lambda}{\operatorname{argmax}} E[W \mid \lambda]=\frac{2 \mathscr{P}_{j, j}-\mathscr{P}_{i, j}-\mathscr{P}_{j, i}}{2\left(\mathscr{P}_{i, i}+\mathscr{P}_{j, j}-\mathscr{P}_{i, j}-\mathscr{P}_{j, i}\right)} \in(0,1) .
$$

This immediately gives us

Proposition 3. Under equal division of rents, the cultural selection condition yields an Utilitarian social welfare optimum.

This follows immediately from noting that under equal division of rents, $\mathscr{P}_{i, j}+\mathscr{P}_{j, i}=$ $\mathscr{E}_{i, j}=\mathscr{E}_{j, i}, 2 \mathscr{P}_{i, i}=\mathscr{E}_{i, i}$ and $2 \mathscr{P}_{j, j}=\mathscr{E}_{j, j}$ so that $(2.6)$ is exactly the same as condition (i) in Proposition 2. Inspection of the complementary slackness conditions also reveals that the welfare optimum is at a corner when the cultural selection involves only one type.

\footnotetext{
${ }^{21}$ Stability here means that after a small perturbation, a dynamic system that satisfies payoff-monotonicity would converge back to the interior equilibrium.

${ }^{22}$ This contrasts with the "Ethnic Enclave" literature in sociology such as Portes and Rumbaut (2001), where economic advantages within a minority ethnic group ensure their continued existence in equilibrium.
} 


\section{Bargaining over Rents}

\subsection{Structure}

We assume now that the division of rents following successful coordination is determined by Nash bargaining, and that the threat points involve receiving an own-type match with an outside option of zero ${ }^{23}$ If we assume that bargaining takes place after the initial match but before the players attempt to coordinate we get

Lemma 1. For an own-type match bargaining over the rents involves

(i) Rents are divided equally; $\alpha^{*}=\frac{1}{2}=\operatorname{argmax} \alpha \mathscr{E}_{k, k}(1-\alpha) \mathscr{E}_{k, k} k \in\{i, j\}$;

(ii) Expected payoffs are $\mathscr{P}_{i, i}=\left(\frac{1}{2}\right) \mathscr{E}_{i, i}$ and $\mathscr{P}_{j, j}=\left(\frac{1}{2}\right) \mathscr{E}_{j, j}$.

Now consider an $i$-type matched with a $j$-type. Employing lemma 1 we get

Lemma 2. For an other-type match bargaining over rents involves

(i) The division of rents satisfies $\beta^{*}=\underset{\beta}{\operatorname{argmax}}\left[\beta \mathscr{E}_{i, j}-\left(\frac{1}{2}\right) \mathscr{E}_{i, i}\right]\left[(1-\beta) \mathscr{E}_{j, i}-\left(\frac{1}{2}\right) \mathscr{E}_{j, j}\right]$;

(ii) With $\beta^{*}=\frac{1}{2}+\frac{\mathscr{E}_{i, i}-\mathscr{E}_{j, j}}{4 \mathscr{E}_{j, i}}$;

(iii) The expected payoffs are $\mathscr{P}_{i, j}=\frac{\mathscr{E}_{i, j}}{2}+\frac{\mathscr{E}_{i, i}-\mathscr{E}_{j, j}}{4}$ and $\mathscr{P}_{j, i}=\frac{\mathscr{E}_{j, i}}{2}+\frac{\mathscr{E}_{j, j}-\mathscr{E}_{i, i}}{4}$.

Notice that part (ii) of Lemma 2 implies that $\beta^{*} \lesseqgtr \frac{1}{2}$ as $\mathscr{E}_{i, i} \lesseqgtr \mathscr{E}_{j, j}$. So, without loss of generality, we hereafter assume that $\mathscr{E}_{i, i}>\mathscr{E}_{j, j}$. If $\frac{\mathscr{E}_{i, i}}{2}>\left(1-\beta^{*}\right) \mathscr{E}_{j, i}$, then $\lambda=1$ is the unique stable equilibrium. In other words, when the rent obtained by an $j$-type agent from an other-type match is too low because of a low outside option in bargaining, then the $j$-type would be assimilated (and similarly an $i$-type). Therefore, to ensure the existence of a stable interior equilibrium, we make the following assumption

Assumption 4. $3 \mathscr{E}_{i, i}-\mathscr{E}_{j, j}<2 \mathscr{E}_{j, i}$, which ensures the existence of a stable interior equilibrium when the division of rents is determined by Nash bargaining.

\footnotetext{
${ }^{23}$ We have in mind the story that each agent may choose to remain at home and play the coordination game with an own-type family member or near neighbor, or engage in matching at a given cost. Hence the expected value of staying at home or engaging in matching will be equalized. Those that choose to engage in matching treat the matching cost as sunk and may return home to an own type match if they choose. This makes the expected value of an own type match the appropriate threat point for bargaining after matching. Then to simplify the exposition we drive the cost of matching to zero and get the analysis presented above.
} 
Assumption 4 also implies a positive bargaining surplus. Notice that for the interior equilibrium we have

$$
\frac{\partial \beta^{*}}{\partial \mathscr{E}_{i, i}}=\frac{1}{4 \mathscr{E}_{j, i}}>0, \quad \frac{\partial \beta^{*}}{\partial \mathscr{E}_{j, j}}=-\frac{1}{4 \mathscr{E}_{j, i}}<0,
$$

implying that an agent's share of rents in an other-type match is increasing in their threat point.

Next we use the values of $\alpha^{*}$ and $\beta^{*}$ to compute the equilibrium value of $\lambda_{b}$, the proportion of $i$-types in the population when rent division is determined by Nash bargaining.

The expected payoffs for the two types are

$$
\begin{aligned}
& E[P(i) \mid \lambda]=\left(\frac{\lambda_{b}}{2}\right) \mathscr{E}_{i, i}+\left(1-\lambda_{b}\right) \beta^{*} \mathscr{E}_{i, j} \\
& E[P(j) \mid \lambda]=\lambda_{b}\left(1-\beta^{*}\right) \mathscr{E}_{j, i}+\left(\frac{1-\lambda_{b}}{2}\right) \mathscr{E}_{j, j} .
\end{aligned}
$$

These expressions together with the cultural selection condition allow us to compute $\lambda_{b}$.

\subsection{Cultural Selection with Bargaining over Rents}

As before, in equilibrium, the cultural selection condition (as defined in Proposition 1) implies that agents choose their types so as to arbitrage the expected returns to types, that is $E[P(i) \mid \lambda]=E[P(j) \mid \lambda]$ employing (3.2) and (3.3) this gives us

$$
\lambda_{b}=\frac{\mathscr{E}_{j, j}-2 \beta^{*} \mathscr{E}_{i, j}}{\mathscr{E}_{i, i}+\mathscr{E}_{j, j}-2 \mathscr{E}_{j, i}}
$$

Using (3.4) and the value of $\beta^{*}$ from Lemma 2 we get

$$
\lambda_{b}=\frac{3 \mathscr{E}_{j, j}-2 \mathscr{E}_{i, j}-\mathscr{E}_{i, i}}{2 \mathscr{E}_{i, i}+2 \mathscr{E}_{j, j}-4 \mathscr{E}_{j, i}}=\frac{1}{2}+\frac{\mathscr{E}_{j, j}-\mathscr{E}_{i, i}}{\mathscr{E}_{i, i}+\mathscr{E}_{j, j}-2 \mathscr{E}_{j, i}} .
$$

Expression (3.5) immediately yields

Proposition 4. $\mathscr{E}_{i, i}>\mathscr{E}_{j, j} \Rightarrow \lambda_{b}>\frac{1}{2}$. That is, the $j$-type is the minority,

Intuitively the group with the lesser threat point benefits less from an other-type match, 
but other-type matches are still better than own-type matches for both types. For the cultural arbitrage condition to hold it must therefore be the case that the group with the lesser threat point are more likely to achieve an other-type match. They must, therefore, be in the minority. We also note that

Proposition 5. $\mathscr{E}_{i, i}>\mathscr{E}_{j, j} \Rightarrow \frac{\partial \beta}{\partial \mathscr{E}_{i, j}}<0$. That is, the minority group benefits disproportionately from an increase in the value of a successful other-type match.

From the welfare optimum (2.6), and employing (3.5) we get

$$
\lambda_{b}=\lambda^{*}+\frac{\mathscr{E}_{j, j}-\mathscr{E}_{i, i}}{2 \mathscr{E}_{i, i}+2 \mathscr{E}_{j, j}-4 \mathscr{E}_{i, j}}
$$

From which we can deduce

Proposition 6. $\mathscr{E}_{i, i}>\mathscr{E}_{j, j} \Rightarrow \lambda_{b}>\lambda^{*}$. That is, the minority group is disproportionately small relative to its size at the social optimum.

When an agent decides to be a member of the minority they only consider the effect this has on their own expected payoffs. However, a social planner in allocating an agent to be of the minority type will consider the effect this has on the probability of an other-type for both of the players matched; hence, Proposition 6.

\subsection{Comparative Statics}

For the interior equilibrium we have

$$
\begin{aligned}
\frac{\partial \lambda_{b}}{\partial \mathscr{E}_{i, i}} & =\frac{2\left(\mathscr{E}_{i, j}-\mathscr{E}_{j, j}\right)}{\left(\mathscr{E}_{i, i}+\mathscr{E}_{j, j}-2 \mathscr{E}_{i, j}\right)^{2}}>0, \\
\frac{\partial \lambda_{b}}{\partial \mathscr{E}_{j, j}} & =\frac{2\left(\mathscr{E}_{i, i}-\mathscr{E}_{i, j}\right)}{\left(\mathscr{E}_{i, i}+\mathscr{E}_{j, j}-2 \mathscr{E}_{i, j}\right)^{2}}<0, \\
\frac{\partial \lambda_{b}}{\partial \mathscr{E}_{i, j}} & =\frac{2\left(\mathscr{E}_{j, j}-\mathscr{E}_{i, i}\right)}{\left(\mathscr{E}_{i, i}+\mathscr{E}_{j, j}-2 \mathscr{E}_{i, j}\right)^{2}}<0 .
\end{aligned}
$$

The first two comparative statics results are obvious, an improvement in a group's threat point increases its equilibrium size. The last comparative statics result is more interesting. It says that if the expected value of a other-type match increases, then this disproportionately 
benefits the group with the lower threat point and hence increases their equilibrium size. This counter-intuitive result follows from the fact that the group with the lesser threat point will be the minority group who are more likely to achieve an other-type match. Hence, the minority is more likely to benefit from an increase in $\mathscr{E}_{i, j}$.

\section{Implications of Policy and Institutions}

The most obvious policy possibilities are to allow a government to engage in taxes/subsidies to effect the rents, the $R(., .)^{\prime} s$, or to facilitate coordination so as to effect the probabilities, the $\rho^{\prime} s$. We have then to consider the circumstances under which the taxes/subsidies are paid and their structure. There are two ways we might approach these issues: First, we might ask what an optimal tax/transfer structure might look like. Then, we might try certain "standard" tax structures and ask what their implications might be, whether they can at least realize Pareto improvements or perhaps implement a welfare optimum.

\subsection{Welfare Optimal Taxes}

If we allow for lump-sum transfers between types but retain the rest of the economic structure of uniformly random matching and Nash bargaining over the rents generated by productive matches. Then a utilitarian social planner will choose a pair of type-specific lump-sum taxes/transfers so as to maximize

$$
\begin{aligned}
& \operatorname{Max}_{T_{i}, T_{j}} \lambda E[P(i) \mid \lambda]+(1-\lambda) E[P(j) \mid \lambda] \\
& \quad=\lambda\left[\lambda\left(\frac{\mathscr{E}_{i, i}}{2}\right)+(1-\lambda) \beta^{*} \mathscr{E}_{i, j}+T_{i}\right]+(1-\lambda)\left[(1-\lambda)\left(\frac{\mathscr{E}_{j, j}}{2}\right)+\lambda\left(1-\beta^{*}\right) \mathscr{E}_{i, j}+T_{j}\right], \\
& \quad \text { s.t. } \lambda T_{i}+(1-\lambda) T_{j}=0, \\
& \quad \lambda=\lambda_{b}+2 \psi\left[T_{j}-T_{i}\right],
\end{aligned}
$$

where $T_{i}, T_{j}$ denote the lump-sum taxes/transfers to/from groups $i, j$. The first part of (4.1) is the utilitarian social welfare function, the second part is the balanced budget constraint, and the third part is a version of the cultural selection condition including the taxes/transfers 
with $\psi \equiv\left[\mathscr{E}_{i, i}+\mathscr{E}_{j, j}-2 \mathscr{E}_{i, j}\right]^{-1}$. Notice first that the balanced budget condition allows the problem to be simplified to

$$
\begin{aligned}
\operatorname{Max}_{T_{i}, T_{j}} & \lambda E[P(i) \mid \lambda]+(1-\lambda) E[P(i) \mid \lambda] \\
& =\lambda\left[\lambda\left(\frac{\mathscr{E}_{i, i}}{2}\right)+(1-\lambda) \beta^{*} \mathscr{E}_{i, j}\right]+(1-\lambda)\left[(1-\lambda)\left(\frac{\mathscr{E}_{j, j}}{2}\right)+\lambda\left(1-\beta^{*}\right) \mathscr{E}_{i, j}\right], \\
& \lambda=\lambda_{b}+2 \psi\left[T_{j}-T_{i}\right] .
\end{aligned}
$$

Furthermore the cultural selection condition may be written as $\lambda=\lambda\left(T_{i}, T_{j}\right)$ so finally the planner's optimization problem reduces to

$$
\begin{aligned}
& \operatorname{Max}_{T_{i}, T_{j}} \lambda\left(T_{i}, T_{j}\right)\left[\lambda\left(T_{i}, T_{j}\right)\left(\frac{\mathscr{E}_{i, i}}{2}\right)+\left(1-\lambda\left(T_{i}, T_{j}\right)\right) \beta^{*} \mathscr{E}_{i, j}\right] \\
& \quad+\left(1-\lambda\left(T_{i}, T_{j}\right)\right)\left[\left(1-\lambda\left(T_{i}, T_{j}\right)\right)\left(\frac{\mathscr{E}_{j, j}}{2}\right)+\lambda\left(T_{i}, T_{j}\right)\left(1-\beta^{*}\right) \mathscr{E}_{i, j}\right] .
\end{aligned}
$$

By solving the planner's problem, we obtain the optimal tax/transfer structure:

$$
\begin{array}{r}
T_{i}=\left(1-\lambda^{*}\right)\left(\frac{1}{2}-\beta^{*}\right) \mathscr{E}_{i j} \\
T_{j}=\lambda^{*}\left(\beta^{*}-\frac{1}{2}\right) \mathscr{E}_{i j}
\end{array}
$$

Plugging the optimal tax/transfer structure into the cultural selection condition we obtain the cultural equilibrium that the social planner aims to induce (denoted by $\left.\lambda_{S P}\right)$ :

$$
\lambda_{S P}=\lambda\left(T_{i}, T_{j}\right)=\lambda^{*}<\lambda_{b}
$$

One can observe that the planner taxes the majority an amount equal to the extra rent that they extract from bargaining and subsidizes the minority with an amount equal to the rent that they lose in bargaining. By doing so, the planner is able to induce the socially optimal cultural equilibrium we derived in Section 2. 


\subsection{Democratically Determined Taxes}

Of greater interest are the effects that actual tax systems have on $\lambda_{b}$, if we assume that the taxes/transfers are determined by democratic voting then as the median voter will be a member of the majority group we assume that taxes are chosen so as to maximize

$$
\begin{gathered}
\operatorname{Max}_{T_{i}, T_{j}} E[P(i) \mid \lambda]=\lambda\left(\frac{\mathscr{E}_{i, i}}{2}\right)+(1-\lambda) \beta^{*} \mathscr{E}_{i, j}+T_{i} \\
\quad \text { s.t. } \lambda T_{i}+(1-\lambda) T_{j}=0 \\
\quad \lambda=\lambda_{b}+2 \psi\left[T_{j}-T_{i}\right] .
\end{gathered}
$$

The first part of (4.7) is the expected payoff of a majority type, the constraints are as in the planners problem. By substitution, (4.7) reduces to

$$
\begin{aligned}
& \operatorname{Max}_{T_{i}, T_{j}} E[P(i) \lambda]=\left(\lambda_{b}+2 \psi\left(T_{j}-T_{i}\right)\right)\left(\frac{\mathscr{E}_{i, i}}{2}\right)+\left[1-\lambda_{b}-2 \psi\left(T_{j}-T_{i}\right)\right] \beta^{*} \mathscr{E}_{i, j}+T_{i}, \\
& \text { s.t. }\left[\lambda_{b}+2 \psi\left(T_{j}-T_{i}\right)\right] T_{i}+\left[1-\lambda_{b}-2 \psi\left(T_{j}-T_{i}\right)\right] T_{j}=0 .
\end{aligned}
$$

If we let $\eta$ be the Lagrangian multiplier associated with the balance budget constraint, we may write the first order conditions as

$$
\begin{aligned}
& \frac{\partial E[P(i) \mid \lambda]}{\partial T_{i}}=-\psi \mathscr{E}_{i, i}+2 \psi \beta^{*} \mathscr{E}_{i, j}+1+\eta\left[\lambda_{b}+2 \psi\left(T_{j}-T_{i}\right)\right]-2 \eta \psi T_{i}+2 \eta \psi T_{j}=0 ; \\
& \frac{\partial E[P(i) \mid \lambda]}{\partial T_{j}}=\psi \mathscr{E}_{i, i}-2 \psi \beta^{*} \mathscr{E}_{i, j}+2 \eta \psi T_{i}+\eta\left[1-\lambda_{b}-2 \psi\left(T_{j}-T_{i}\right)\right]-2 \eta \psi T_{j}=0 .
\end{aligned}
$$

Eliminating the multiplier from conditions (4.9) and (4.10) gives

$$
T_{j}-T_{i}=\frac{\mathscr{E}_{i i}-\mathscr{E}_{j j}}{4}>0
$$

Hence, the majority would vote to subsidize the minority. By plugging $T_{j}-T_{i}$ into the cultural selection condition, we obtain the cultural equilibrium that a democratically determined 
tax/transfer structure induces (denoted by $\lambda_{D D}$ ):

$$
\lambda_{D D}=\lambda^{*}
$$

This indicates that the democratically determined tax/transfer structure is identical to the social optimal tax/transfer structure. Both encourage multi-culturalism and induce social optima. ${ }^{24}$ The intuition here is quite straightforward, the cultural selection condition implies that the agents arbitrage the expected payoff to cultural type. In essence the majority internalize the effects of their choices on the minority because of the cultural selection condition in the program (4.7).

\subsection{A Simple Social Welfare System}

Suppose we assume that there is a simple welfare system in which productive matches, those that involve successful coordination, are taxed with the revenues used to pay all citizens a welfare payment, this may be thought of as an uniform proportional income tax used to finance the provision of a service such as universal health care. This may be represented as

$$
\begin{aligned}
\operatorname{Max}_{t, s} & \lambda E[P(i) \mid \lambda]+(1-\lambda) E[P(j) \mid \lambda] \\
& =\lambda\left[\lambda\left(\frac{\mathscr{E}_{i, i}}{2}\right)(1-t)+(1-\lambda) \beta^{*} \mathscr{E}_{i, j}(1-t)+s\right] \\
& +(1-\lambda)\left[(1-\lambda)\left(\frac{\mathscr{E}_{j, j}}{2}\right)(1-t)+\lambda\left(1-\beta^{*}\right) \mathscr{E}_{i, j}(1-t)+s\right], \\
& \text { s.t. } \lambda\left(\frac{\mathscr{E}_{i, i}}{2}\right)(1-t)+(1-\lambda) \beta^{*} \mathscr{E}_{i, j}(1-t)+s \\
& =(1-\lambda)\left(\frac{\mathscr{E}_{j, j}}{2}\right)(1-t)+\lambda\left(1-\beta^{*}\right) \mathscr{E}_{i, j}(1-t)+s, \\
& {\left[\lambda^{2} \mathscr{E}_{i, i}+2 \lambda(1-\lambda) \mathscr{E}_{i, j}+(1-\lambda)^{2} \mathscr{E}_{j, j}\right] t=2 s . }
\end{aligned}
$$

Where the first constraint is the cultural selection condition, and the second is the plan-

\footnotetext{
${ }^{24}$ Classic examples of these policies may be found in the Netherlands which pursued a policy of "pillarization" (multiculturalism) based on the 1983 Ethnic Minorities Policy, but then reversed this policy to one which encouraged integration via the Civic Integration of Newcomers Act of 1998. See Vasta (2007) for details.
} 
ners balanced budget condition where the $2 s$ reflects that the taxes are per match and the subsidy are per person.

The cultural selection condition can be simplified to

$$
\lambda\left(\frac{\mathscr{E}_{i, i}}{2}\right)+(1-\lambda) \beta^{*} \mathscr{E}_{i, j}=(1-\lambda)\left(\frac{\mathscr{E}_{j, j}}{2}\right)+\lambda\left(1-\beta^{*}\right) \mathscr{E}_{i, j}
$$

which is exactly the condition we needed for an interior equilibrium in Section 3.2. This implies that no matter how $t$ and $s$ are specified, the cultural equilibrium is always $\lambda_{b}$. Hence, the welfare program yields the market equilibrium and is not able to effect social welfare.

\section{The Intensity of Social Interaction}

In the preceding sections each agent of each cultural type is allowed only one uniformly random match. Suppose now instead that there are potentially several "rounds" of matching. Individuals in each round may accept or reject the match they obtain. If they accept then the matched pair withdraw from the matching process and immediately play the coordination game, furthermore if they achieve coordination they immediately engage in Nash bargaining over the rents. Those that do not accept matches try again in the next round. The process continues for $n$ rounds, thereafter any agents that are unmatched receive an own-type match as in our previous bargaining game. $n$ therefore is our measure of the intensity of social interaction. We interpret a higher $n$ as greater intensity representing lesser exogenous impediments to social interaction: These may involve the physical geography of the space in which matching takes place, population density, or the residual time available to engage in matching after the time required for coordination, production and bargaining has been allocated.25

\footnotetext{
${ }^{25}$ There appears to be little research concerning the effects of increases in the frequency with which agents interact on the productivity of those interactions, however we note that the effect of more frequent interactions, if own-type matches are rejected, is equivalent in terms of the effect on the expected value of a match to a greater number of minority types involved in the matching process. There is a great deal of evidence on this in a very wide variety of context, almost all of which points to an increase in productivity. For a recent summary of the evidence see Kemeny (2014), in another paper Kemeny (2012) shows that cultural diversity increases productivity in terms of the wages of native-born workers, and that there is not reverse causality in the sense that these workers demand a wage premium to tolerate diversity
} 
If we assume uniformly random matching, and if the initial proportions of $i$-types and $j$-types are $\lambda$ and $1-\lambda$ respectively then following Hardy(1908) and Boylan (1992) we may write

Proposition 7. If an initial population of unit length consists of $\lambda$-type $i$ 's and $1-\lambda$-type $j$ 's then with uniformly random matching the proportions of $i i, j j$ and ij matches are given by $\lambda^{2},(1-\lambda)^{2}$, and $2 \lambda(1-\lambda)$ respectively.

We shall demonstrate shortly that all cross culturally matched pairs choose to withdraw from further matching and play the coordination game, whereas all own-type matched pairs choose to reject their current matches and try again in the hope of an other-type match. Denote as $\hat{\lambda}_{m}$ the proportion of majority $i$-types in the population still seeking a match after $m$ rounds of matching. That is after those that have achieved an other-type match have withdrawn from the population engaged in the matching process. After $m$ rounds of matching the proportion of types $i$ and $j$ in the population may be written

$$
\hat{\lambda}_{m+1}=\frac{\hat{\lambda}_{m}^{2}}{\hat{\lambda}_{m}^{2}+\left(1-\hat{\lambda}_{m}\right)^{2}} \text { and } 1-\hat{\lambda}_{m+1}=\frac{\left(1-\hat{\lambda}_{m}\right)^{2}}{\hat{\lambda}_{m}^{2}+\left(1-\hat{\lambda}_{m}\right)^{2}}
$$

Hence the proportions of the two types in the population follow a first order non-linear difference equation. Notice that the population is in a steady state at $\lambda=\left\{0, \frac{1}{2}, 1\right\}$. Further by successive substitution we may obtain the population proportions after $m$ rounds of matching as functions of the initial population proportions to be

$$
\hat{\lambda}_{m}=\frac{\lambda^{2^{m-1}}}{\lambda^{2^{m-1}}+(1-\lambda)^{2^{m-1}}} .
$$

We immediately have

Lemma 3. $\hat{\lambda}_{m}$ is monotonically increasing in $m$ if $\lambda>\frac{1}{2}$.

Lemma 3 tells us that in later rounds of matching minority $j$-types are more likely to achieve an other-type match and majority $i$-types less likely to do so. This plays an important role in the results that follow because it is anticipated by the agents and thus effects their earlier decisions. The number of matching rounds asymmetrically effects the bargaining threat points of the two types and thus, in turn, effects their choices of cultural type. 
We now solve the sequential bargaining process given the social interaction paradigm outlined above. We have assumed that both cultural types reject own-type matches and accept other-type matches in all rounds except the last. Now we demonstrate that these maintained assumptions are correct.

Assume that there are $n$ rounds of social interactions. In the last round, clearly all agents accept their matches and each agent's expected payoff is the same as derived in section 3 except that the population proportions are as given by (5.2) with $m=n$. We can thus write the expected payoffs of the two types of agents in the last round as:

$$
\begin{aligned}
& E_{n}[P(i), n, \lambda]=\hat{\lambda}_{n}\left(\frac{\mathscr{E}_{i, i}}{2}\right)+\left(1-\hat{\lambda}_{n}\right)\left(\frac{\mathscr{E}_{j, i}}{2}+\frac{\mathscr{E}_{i, i}-\mathscr{E}_{j, j}}{4}\right), \\
& E_{n}[P(j), n, \lambda]=\hat{\lambda}_{n}\left(\frac{\mathscr{E}_{j, i}}{2}-\frac{\mathscr{E}_{i, i}-\mathscr{E}_{j, j}}{4}\right)+\left(1-\hat{\lambda}_{n}\right)\left(\frac{\mathscr{E}_{j, j}}{2}\right) .
\end{aligned}
$$

It can easily be verified that $E_{n}[P(i), n, \lambda]>\frac{\mathscr{E}_{i, i}}{2}, E_{n}[P(j), n, \lambda]>\frac{\mathscr{E}_{j, j}}{2}$ and $E_{n}[P(i), n, \hat{\lambda}]+$ $E_{n}[P(j), n, \hat{\lambda}]<\mathscr{E}_{j, i}$.

Next, consider rounds 1 to $n-1$. Let $E_{t}[P(k), n, \lambda]$ with $k \in\{i, j\}$ denote the expected payoff of a $k$-type agent in any round $t \in\{1,2, \ldots, n-1\}$, in any round $t-1$ this also represents the players outside option if they reject their current matches. Further if we denote as $\beta_{t}$ the share of rents obtained by an $i$-type agent in the $t^{t h}$ round for any $t \in\{1,2, \ldots, n-1\}$, then from the analysis of Section 3 we know that $\beta_{t}=\frac{1}{2}+\frac{E_{t+1}[P(i), n, \lambda]-E_{t+1}[P(j), n, \lambda]}{2 \mathscr{E}_{j, i}}$.

We can thus write the expected payoffs of the two type agents in any round for any $t \in\{1,2, \ldots, n-1\}$ as:

$$
\begin{aligned}
E_{t}[P(i), n, \lambda]= & \hat{\lambda}_{t} E_{t+1}[P(i), n, \lambda] \\
& +\left(1-\hat{\lambda}_{t}\right)\left(\frac{\mathscr{E}_{j, i}}{2}+\frac{E_{t+1}[P(i), n, \lambda]-E_{t+1}[P(j), n, \lambda]}{2}\right), \\
E_{t}[P(j), n, \lambda]= & \hat{\lambda}_{t}\left(\frac{\mathscr{E}_{j, i}}{2}-\frac{E_{t+1}[P(i), n, \lambda]-E_{t+1}[P(j), n, \lambda]}{2}\right) \\
& +\left(1-\hat{\lambda}_{t}\right) E_{t+1}[P(i), n, \lambda] .
\end{aligned}
$$


Adding these together gives us

$$
E_{t}[P(i), n, \lambda]+E_{t}[P(j), n, \lambda]=\frac{\mathscr{E}_{j, i}}{2}+\frac{E_{t+1}[P(i), n, \lambda]+E_{t+1}[P(j), n, \lambda]}{2},
$$

for any $t \in\{1,2, \ldots, n-1\}$.

Now given that $E_{n}[P(i), n, \lambda]+E_{n}[P(j), n, \lambda]=\frac{\mathscr{E}_{j, i}}{2}+\frac{1}{4}\left(\mathscr{E}_{i, i}+\mathscr{E}_{j, j}\right)$, successive substitutions yield

$$
\begin{aligned}
E_{t}[P(i), n, \lambda]+E_{t}[P(j), n, \lambda] & =\frac{2^{n+1-t}-1}{2^{n-t}}\left(\frac{\mathscr{E}_{j, i}}{2}\right)+\frac{1}{2^{n+1-t}}\left(\frac{\mathscr{E}_{i, i}+\mathscr{E}_{j, j}}{2}\right) \\
& =\mathscr{E}_{j, i}-\frac{1}{2^{n+1-t}}\left(\mathscr{E}_{j, i}-\frac{\mathscr{E}_{i, i}}{2}-\frac{\mathscr{E}_{j, j}}{2}\right)<\mathscr{E}_{j, i} .
\end{aligned}
$$

for any $t \in\{1,2, \ldots, n-1\}$. The left hand side of $(5.8)$ is the sum of expected values of the two players outside options while the right hand side is the surplus to be split given they achieve an other-type match. It is then immediate that other-type matches are accepted and the players engage in Nash bargaining. Now we demonstrate that own-type matches are rejected. Again employing (5.5) and (5.6) we may write

$$
\begin{aligned}
E_{t-1}[P(i), n, \lambda] & -E_{t}[P(i), n, \lambda] \\
& =\left(1-\hat{\lambda}_{t-1}\right)\left(\frac{\mathscr{E}_{j, i}}{2}-\frac{1}{2}\left(E_{t}[P(i), n, \lambda]+E_{t}[P(j), n, \lambda]\right)\right), \\
E_{t-1}[P(j), n, \lambda] & -E_{t}[P(j), n, \lambda] \\
& =\hat{\lambda}_{t-1}\left(\frac{\mathscr{E}_{j, i}}{2}-\frac{1}{2}\left(E_{t}[P(i), n, \lambda]+E_{t}[P(j), n, \lambda]\right)\right),
\end{aligned}
$$

for any $t \in\{2, \ldots, n\}$.

Now since the right hand sides of (5.9) and (5.10) are positive it follows that $E_{t}[P(i), n, \lambda]$ and $E_{t}[P(j), n, \lambda]$ are both monotonically decreasing in $t$. Since $E_{n}[P(i), n, \lambda]>\frac{\mathscr{E}_{i, i}}{2}$ and $E_{n}[P(j), n, \lambda]>\frac{\mathscr{E}_{j, j}}{2}$, we have $E_{t}[P(i), n, \lambda]>\frac{\mathscr{E}_{i, i}}{2}$ and $E_{t}[P(j), n, \lambda]>\frac{\mathscr{E}_{j, j}}{2}$ for any $t \in$ $\{1,2, \ldots, n-1\}$. Therefore the assumption that for all but the last round own-type matches are rejected is satisfied. Thus we have shown that the social interaction paradigm hypothesized in (5.2) is correct given the sequence of matching decisions and bargaining outcomes. We 
thus have a unique equilibrium solution to the sequential matching/bargaining problem.

Using (5.2)-(5.6), we can derive the expected payoffs of the two types of agents as shown in the following proposition:

Proposition 8. The expected payoffs of the two types of agents derived from the unique equilibrium of the sequential bargaining problem are given by

$$
\begin{aligned}
& E_{1}[P(i), n, \lambda]=\frac{\mathscr{E}_{i, i}}{2}+\left(\mathscr{E}_{j, i}-\frac{\mathscr{E}_{i, i}}{2}-\frac{\mathscr{E}_{j, j}}{2}\right) \sum_{t=1}^{n} \frac{1-\hat{\lambda}_{t}}{2^{n+1-t}}, \\
& E_{1}[P(j), n, \lambda]=\frac{\mathscr{E}_{j, j}}{2}+\left(\mathscr{E}_{j, i}-\frac{\mathscr{E}_{i, i}}{2}-\frac{\mathscr{E}_{j, j}}{2}\right) \sum_{t=1}^{n} \frac{\hat{\lambda}_{t}}{2^{n+1-t}},
\end{aligned}
$$

where $\hat{\lambda}_{t}$ obeys (5.2).

Proposition 8 has the following immediate implications:

Corollary 1. $\lambda=\frac{1}{2}$ is not an equilibrium for any $n$.

Proof: When $\lambda=\frac{1}{2}, \hat{\lambda}_{t}=\frac{1}{2}$ for any $t>0$. Therefore, we have $E_{1}\left[P(i), n, \frac{1}{2}\right]-E_{1}\left[P(j), n, \frac{1}{2}\right]=$ $\frac{\mathscr{E}_{i, i}}{2}-\frac{\mathscr{E}_{j, j}}{2}>0$, for any $n$. But this violates the cultural selection condition and hence cannot hold. Q.E.D.

Corollary 1 indicates that $j$-type agents remain as a minority regardless of the number of rounds of social interactions. To gain some intuition into this result suppose that there were equal numbers of the two types, and that there were sufficient rounds of social interaction such that all agents found an other-type match. Then each match would involve the same surplus to be divided, but given the $i$-types have a higher threat point they must capture a relatively larger share of the surplus, but this cannot be consistent with two types being equal in numbers

Corollary 2. There exists an $\underline{n}>0$ such that for any $n>\underline{n}, \lambda=1$ is not an equilibrium.

Proof: We have $\lim _{\lambda \rightarrow 1} \hat{\lambda}_{t}=1$. Hence, $\lim _{\lambda \rightarrow 1} E_{1}[P(i), n, \lambda]=\frac{\mathscr{E}_{i, i}}{2}, \lim _{\lambda \rightarrow 1} E_{1}[P(j), n, \lambda]=$ $\frac{\mathscr{E}_{j, j}}{2}+\left(\mathscr{E}_{j, i}-\frac{\mathscr{E}_{i, i}}{2}-\frac{\mathscr{E}_{j, j}}{2}\right) \sum_{t=1}^{n} \frac{1}{2^{n+1-t}}$. When $n$ approaches infinity, we have $\lim _{n \rightarrow \infty} \lim _{\lambda \rightarrow 1} E_{1}$ $[P(j), n, \lambda]=\mathscr{E}_{j, i}-\frac{\mathscr{E}_{i, i}}{2}>\frac{\mathscr{E}_{i, i}}{2}$. Therefore, there exists an $\underline{n}>0$, such that for any $n>\underline{n}$, $\lambda=1$ is not a equilibrium. Q.E.D. 
Recall that in Section 3, we showed that it is possible that all $j$-type agents get assimilated if the rents they extract from bargaining are too low. However, Corollary 2 indicates that this is no longer the possible when social interactions are sufficiently intense. In other words, intense social interactions help to preserve cultural heterogeneity 26 Intuitively this follows from (5.1), more rounds of matching both increase the probability that minority $j$-types will realize valuable other-type matches and raise the minority types threat points in the sequential bargaining process. It follows that with enough rounds of social interaction some agents will always choose to be $j$-types. We now turn to the relationship between the intensity of social interaction and the size of the minority group.

Since $\hat{\lambda}_{t}$ monotonically increases in $\lambda$ for any $t>0,(5.10)$ and (5.11) indicate that $E_{1}[P(i), n, \lambda]$ is strictly decreasing in $\lambda$ and $E_{1}[P(j), n, \lambda]$ is strictly increasing in $\lambda$. Combining this with Corollaries 1 and 2, immediately implies that there exists a unique stable interior equilibrium in $\left(\frac{1}{2}, 1\right)$ when $n>\underline{n}$. Let $\lambda^{*}(n)$ denote the interior equilibrium for $n>\underline{n}$. We have the following result:

Corollary 3. $\lambda^{*}(n)$ monotonically decreases in $n$ for $n>\underline{n}$.

Proof: See Appendix.

Corollary 3 indicates that intense social interactions help to increase the size of the minority group. The intuition here is that more intense social interaction increases the relative probability that the minority will find an other-type match. Since other-type matches have higher expected payoffs and since unmatched j-types become relatively scarcer as the number of matching rounds increases, then it follows that j-types capture more of the rents from each other-type match. Hence in equilibrium more agents choose to be j-types the greater is social interaction. This runs counter to the casual intuition that greater exposure of the minority type to the majority type might induce greater assimilation.

Next, we consider social welfare at the interior equilibrium. We are interested in the question of whether greater social interaction is welfare improving and indeed if it is Pareto improving? By Corollary 2, we know that when $n \leq \underline{n}$ (if $\underline{n} \geq 1$ ), $\lambda=1$ is the unique

\footnotetext{
${ }^{26}$ In the Ethnic Studies and Sociology literature the distinction is drawn between cultural maintenance and cultural adaption, where the latter is sometimes interpreted as the intensity of cultural interaction. The question often studied is whether the two are substitutes or complements. See for example Verkuyten and Thijs (2002) who study the Turkish minority in the Netherlands. Here the two are complements.
} 
equilibrium. Hence, social welfare equals $\frac{\mathscr{E}_{i, i}}{2}$. On the other hand, when $n>\underline{n}$, we have the interior equilibrium $\lambda^{*}(n)$. Let $E\left[W, n, \lambda^{*}(n)\right]$ denote the Utilitarian social welfare in the interior equilibrium for $n>\underline{n}$, we have the following result:

Proposition 9. For $n>\underline{n}$, to maximize social welfare the planner maximizes the utility of either type of agent, that is $E\left[W, n, \lambda^{*}(n)\right]=E_{1}\left[P(k), n, \lambda^{*}(n)\right]=\frac{\mathscr{E}_{j, i}}{2}-\frac{\mathscr{E}_{i, i}+\mathscr{E}_{j, j}}{2^{n+2}} k=i, j$.

Proof: (5.10) and (5.11) imply that

$$
E_{1}\left[P(i), n, \lambda^{*}(n)\right]+E_{1}\left[P(j), n, \lambda^{*}(n)\right]=\mathscr{E}_{j, i}-\frac{\mathscr{E}_{i, i}+\mathscr{E}_{j, j}}{2^{n+1}}
$$

Since in state $\lambda^{*}(n), E_{1}\left[P(i), n, \lambda^{*}(n)\right]=E_{1}\left[P(j), n, \lambda^{*}(n)\right]$, we have

$$
\begin{aligned}
E\left[W, n, \lambda^{*}(n)\right] & =\lambda^{*}(n) E_{1}\left[P(i), n, \lambda^{*}(n)\right]+\left(1-\lambda^{*}(n)\right) E_{1}\left[P(j), n, \lambda^{*}(n)\right] \\
& =E_{1}\left[P(i), n, \lambda^{*}(n)\right]=\frac{\mathscr{E}_{j, i}}{2}-\frac{\mathscr{E}_{i, i}+\mathscr{E}_{j, j}}{2^{n+2}} .
\end{aligned}
$$

Q.E.D.

Proposition 9 first indicates that, for $n>\underline{n}, E\left[W, n, \lambda^{*}(n)\right]$ increases in $n$. In other words, intensive social interactions benefit the whole society. Second, we have $\lim _{n \rightarrow \infty} E\left[W, n, \lambda^{*}(n)\right]$ $=\frac{\mathscr{E}_{j, i}}{2}$. Therefore, in the limit more intensive social interactions lead to the Pareto optimum. Note that the social optimum we derived in Section 2, where no bargaining takes place and matching involves only one round, involves $\frac{\left(\frac{\mathscr{E}_{j, i}}{2}\right)^{2}-\frac{\mathscr{E}_{i, i}}{2} \frac{\mathscr{E}_{j, j}}{2}}{\mathscr{E}_{j, i}-\frac{\mathscr{E}_{i, i}}{2}-\frac{\mathscr{E}_{j, j}}{2}}<\frac{\mathscr{E}_{j, i}}{2}$. Hence, increased social interaction can eliminate the inefficiency that arises from bargaining and improve the social welfare.

\section{Dynamics}

Here we provide two dynamic foundations for the cultural selection results we obtained in a static environment in previous sections. We first investigate an overlapping generation model in which each parent can directly choose their child's culture type subject to an 
increasing convex cost function in the number of cross-cultural shifts. Second, we examine a probabilistic cultural transmission model based on Bisin and Verdier (2001).

\subsection{Increasing Convex Cost Function}

Consider a overlapping generations model. Each agent lives for two periods, young and old, and has one child when old. Old agents are altruistic towards their children. Only old agents are economically active, they engage in economic activity as described in Section 3 above, plus they may choose their child's cultural type. If they choose the child's cultural type to be different from their own they face a cost of increasing convex cost function in the number of cross-cultural shifts that occur in their cultural group. This might represent the cost of sending children to a different school or relocating to a different geographic location.

The lifetime utility of a type $i$ agent is now given by

$$
E\left[U_{i} \mid \lambda\right]=E[P(i) \mid \lambda]+E\left[P_{i c} \mid \lambda\right]-\frac{\tau_{i}^{2}}{2 \psi_{i}},
$$

which is the sum of the agents own expected payoff and the expected payoff of the child $\left(E\left[P_{i c} \mid \lambda\right]\right)$ minus a cost term. The expected earnings of the child equals either $E[P(i) \mid \lambda]$ or $E[P(j) \mid \lambda]$. The cost term is a quadratic function of the number of $i$-type agents who indoctrinate their children to become $j$-types in the period $\left(\tau_{i}\right)$. In addition, the cost term is effected by, $\psi_{i}$, the "environment" in which the indoctrination takes place. For a majority $i$-type we write the environment as

$$
\psi_{i}=\left(\rho_{i, i}\right)^{\sigma_{i}}\left(\rho_{i, j}\right)^{\zeta_{i}}
$$

with parameters $\sigma_{i} \in(-1,0)$ and $\zeta_{i} \in(0,1)$. A larger $\rho_{i, i}$ implies a smaller $\psi_{i}$ and makes cross-cultural shifts more costly. In this context we interpret $\rho_{i, i}$ as the cohesion of the majority group and argue that greater cohesiveness leads to more indirect indoctrination from friends, family, and neighbors making it more difficult and costly for a parent to successfully change a child's cultural type. A larger $\rho_{i, j}$ implies that the cultural distance between the two groups is smaller making it less costly for a parent to successfully change a child's cultural 
type. $\sigma_{i}$ and $\zeta_{i}$ determine the relative importance of the local environment, and the more general environment for the cost of cross-cultural shifts. ${ }^{27}$

The lifetime utility of a type $j$ agent takes the same form:

$$
E\left[U_{j} \mid \lambda\right]=E[P(j) \mid \lambda]+E\left[P_{j c} \mid \lambda\right]-\frac{\tau_{j}^{2}}{2 \psi_{j}},
$$

where $E\left[P_{j c} \mid \lambda\right]=E[P(i) \mid \lambda]$ or $E[P(j) \mid \lambda]$ is the expected earnings of the child and $\tau_{j}$ denotes the number of type $j$ agents who indoctrinate their children to become type $i$.

An agent would choose a different cultural type for a child if the marginal increment to expected earnings of the child exceeds the marginal cost. The following lemma summarizes the equilibrium number of cross-cultural shifts in the population:

Lemma 4. If $E[P(j) \mid \lambda]>E[P(i) \mid \lambda], \tau_{i}^{*}=\psi_{i}(E[P(j) \mid \lambda]-E[P(i) \mid \lambda]), \tau_{j}^{*}=0$; if $E[P(j) \mid \lambda]<$ $[P(i)], \tau_{j}^{*}=\psi_{j}(E[P(i) \mid \lambda]-E[P(j) \mid \lambda]), \tau_{i}^{*}=0$; if $E[P(i) \mid \lambda]=E[P(j) \mid \lambda], \tau_{i}^{*}=\tau_{j}^{*}=0$.

By adding time indexes to all variables, we can describe the dynamics of cultural selection by the following difference equation:

$\lambda_{t+1}= \begin{cases}\max \left\{\lambda_{t}-\tau_{i t}^{*}, 0\right\} & \text { if } E_{t}\left[P(i) \mid \lambda_{t}\right]<E_{t}\left[P(j) \mid \lambda_{t}\right], \\ \min \left\{\lambda_{t}+\tau_{j t}^{*}, 1\right\} & \text { if } E_{t}\left[P(i) \mid \lambda_{t}\right]>E_{t}\left[P(j) \mid \lambda_{t}\right], \\ \lambda_{t} & \text { if } E_{t}\left[P(i) \mid \lambda_{t}\right]=E_{t}\left[P(j) \mid \lambda_{t}\right],\end{cases}$

The cultural selection dynamic satisfies payoff-monotonicity. That is, the cultural group with the higher expected payoff expands in the population over time.

The condition for an interior steady state in the dynamics is $E_{t}[P(i)]=E_{t}[P(j)]$, now from section 3 this implies $\lambda_{t}=\lambda_{t+1}=\lambda_{b}$, so we are concerned with whether the system converges to $\lambda_{b}$. We have

Proposition 10. When $\max \left\{\psi_{i}, \psi_{j}\right\}\left(\frac{\mathscr{E}_{i, i}+\mathscr{E}_{j, j}}{2}-\mathscr{E}_{i, j}\right)>-2, \lambda_{b}$ is globally asymptotically stable. When $\max \left\{\psi_{i}, \psi_{j}\right\}\left(\frac{\mathscr{E}_{i, i}+\mathscr{E}_{j, j}}{2}-\mathscr{E}_{i, j}\right)<-2, \lambda_{b}$ is repelling.

Proof: Note that when the interior equilibrium is stable then the boundary states 0 and 1 are not attracting. Hence, when studying the stability of an interior equilibrium, we can

\footnotetext{
${ }^{27} \mathrm{~A}$ similar definition can be supplied for $\psi_{j}$ but we omit it as all the subsequent results are symmetric.
} 
safely ignore these bounds and rewrite the proportion of the population of type $i$ after $t$ periods given an initial state $\lambda_{0} \in[0,1]$ as

$$
\lambda_{t+1}= \begin{cases}\left(1+\psi_{i}\left(\frac{\mathscr{E}_{i, i}+\mathscr{E}_{j, j}}{2}-\mathscr{E}_{i, j}\right)\right) \lambda_{t}+\psi_{i} \frac{\mathscr{E}_{j, j}}{2}-\beta \mathscr{E}_{i, j} & \text { if } \lambda_{t}>\lambda_{b} \\ \left(1+\psi_{j}\left(\frac{\mathscr{E}_{i, i}+\mathscr{E}_{j, j}}{2}-\mathscr{E}_{i, j}\right)\right) \lambda_{t}+\psi_{j} \frac{\mathscr{E}_{j, j}}{2}-\beta \mathscr{E}_{i, j} & \text { if } \lambda_{t}<\lambda_{b} \\ \lambda_{t} & \text { if } \lambda_{t}=\lambda_{b}\end{cases}
$$

with initial condition $\lambda_{0} \in[0,1]$.

If both $\left|1+\psi_{i}\left(\frac{\mathscr{E}_{i, i}+\mathscr{E}_{j, j}}{2}-\mathscr{E}_{i, j}\right)\right|<1$ and $\left|1+\psi_{j}\left(\frac{\mathscr{E}_{i, i}+\mathscr{E}_{j, j}}{2}-\mathscr{E}_{i, j}\right)\right|<1$, then $\lambda_{b}$ is globally asymptotically stable. If either $\left|1+\psi_{i}\left(\frac{\mathscr{E}_{i, i}+\mathscr{E}_{j, j}}{2}-\mathscr{E}_{i, j}\right)\right|>1$ or $\left|1+\psi_{j}\left(\frac{\mathscr{E}_{i, i}+\mathscr{E}_{j, j}}{2}-\mathscr{E}_{i, j}\right)\right|>1$, then $\lambda_{b}$ is repelling. Q.E.D.

If the condition $\max \left\{\psi_{i}, \psi_{j}\right\}\left(\frac{\mathscr{E}_{i, i}+\mathscr{E}_{j, j}}{2}-\mathscr{E}_{i, j}\right)<-2$ for instability in Proposition 10 is satisfied, the system displays oscillatory divergence. So, in the long run, $\lambda_{t} \in\{0,1\}$ with the system bouncing between two homogeneous states. Hence, although other-type interaction is beneficial to the society, it is possible that cultural heterogeneity fails to emerge as a stable equilibrium. Notice that this result holds when the environment in which socialization takes place is conducive to cultural change, that is $\max \left\{\psi_{i}, \psi_{j}\right\}$ is sufficiently large. This arises when the "cultural distance" between the two groups is sufficiently small (sufficiently large $\rho_{i j}$ ) and/or when within group cohesion is low (sufficiently low $\rho_{i, i}$ or $\rho_{j, j}$ ) making it inexpensive for the agents to switch cultures, and when the other-type interaction payoff $\mathscr{E}_{i, j}$ is large compared to the own-type interaction payoffs $\mathscr{E}_{i, i}$ and $\mathscr{E}_{j, j}$. Casually it might appear that this relationship between the expected payoffs from the different matches would tend to make the system stable. This is not so. The key problem is that each individual when choosing the cultural type of their child assumes that their switch has no measurable effect on the proportions of the types in the population. Suppose, for example, that all agents are type $i$ then each believes that switching their child to a type $j$ will ensure a high payoff other-type match for their child, but as they all behave this way the system oscillates and the populaton is always of one type! Furthermore a high $\mathscr{E}_{i, j}$ relative to $\mathscr{E}_{i, i}$ and $\mathscr{E}_{j, j}$ simply 
reinforces this behavior. We do not intend this result to be taken literally as a description of any real economy, but do regard it as indicative of the sort of inefficiencies that the dynamic path can display as a consequence of agents not internalizing the effects of their own cultural choices on the expected payoffs of others.

Now let us consider the social planner's problem. The planner wishes to find a mechanism to implement the welfare optimum $\lambda^{*}$, and for the path to that optimum to be efficient given the adjustment costs.

The first obvious instrument that the social planner can employ is a tax scheme that "corrects" the agents' incentives and enables the dynamics to converge to the socially optimal equilibrium $\lambda^{*}$. However, the social planner needs to take into consideration whether the socially optimal equilibrium is stable. The following result supplies a balanced budget tax scheme that gives $\lambda^{*}$ as the interior equilibrium and then considers the stability of that equilibrium.

Proposition 11. When the balanced budget tax scheme involves that in each generation $t$ the planner taxes each $i$-type agent an amount equal to $T_{i t}=\left(1-\lambda_{t}\right)\left(\beta-\frac{1}{2}\right) \mathscr{E}_{i j}$ and taxes each $j$-type agent an amount equal to $T_{j t}=-\lambda_{t}\left(\beta-\frac{1}{2}\right) \mathscr{E}_{i j}$, then the interior equilibrium is $\lambda^{*}$. Further, when $\max \left\{\psi_{i}, \psi_{j}\right\}\left(\frac{\mathscr{E}_{i, i}+\mathscr{E}_{j, j}}{2}-\mathscr{E}_{i, j}\right)>-2$, the equilibrium is globally asymptotically stable given the tax scheme. But when $\max \left\{\psi_{i}, \psi_{j}\right\}\left(\frac{\mathscr{E}_{i, i}+\mathscr{E}_{j, j}}{2}-\mathscr{E}_{i, j}\right)<-2, \lambda^{*}$ is repelling given the tax scheme.

Proof: See Appendix.

The lesson we learn from Proposition 11 is that the condition for stability of $\lambda^{*}$ is exactly the same as that for $\lambda_{b}$. Hence, if $\lambda_{b}$ is stable, the social planner can implement the tax scheme $T_{i t}$ and $T_{j t}$ to induce a stable socially optimal state.

On the other hand, if $\lambda_{b}$ is repelling, the social planner strictly prefers a stable homogeneous state $\lambda=1$ to a unstable population state switching between 0 and 1 . This alternating behavior yields maximal costs of cross-cultural shifts in every period, without realizing any beneficial other-type matches. Hence, the social planner should employ a tax scheme that induces monotonic convergence to $\lambda=1$.

The balanced budget tax scheme described above implements the welfare optimum, $\lambda^{*}$, 
and is stable if the economy is stable in the absence of these taxes. However, it does not guarantee that the transition path is optimal. We examine this issue next. To simplify the exposition, we now allow the social planner to directly manipulate the cultural selection process but we can easily back out the necessary tax rates. Hence the transitional dynamics from any initial condition, $\lambda_{0}$ to the terminal condition, $\lambda^{*}$ are chosen by the planner to be welfare optimal. Without loss of generality assume $\lambda_{0}>\lambda^{*}$. Let $s_{t}$ denote the number of $i$-type agents whose children the social planner chooses to convert to $j$-types at time t. Hence, $\lambda_{t+1}=\lambda_{t}-s_{t}$. The social planner solves the following optimization problem:

$$
\begin{aligned}
\max & \sum_{t=0}^{\infty}\left(\lambda_{t+1} E_{t+1}\left[P(i) \mid \lambda_{t+1}\right]+\left(1-\lambda_{t+1}\right) E_{t+1}\left[P(j) \mid \lambda_{t+1}\right]-\frac{s_{t}^{3}}{2 \psi_{i}}\right) \\
& \text { such that } \lambda_{t+1}=\lambda_{t}-s_{t} .
\end{aligned}
$$

In other words, the social planner maximizes the aggregate welfare of the agents (including the costs experienced by the $i$-type agents whose children are converted to $j$-types) by choosing the step sizes $\left(s_{t}\right)$ and the number of steps to reach $\lambda^{*}$ from $\lambda_{0}$.

The dynamic optimization problem faced by the social planner can be approximated by the following linear-quadratic regulator (Bertsekas (1995), Hansen and Sargent (2007)) with instantaneous return equal to

$$
-\frac{2 \mathscr{E}_{i j}-\mathscr{E}_{i i}-\mathscr{E}_{j j}}{2}\left(\lambda_{t}-s_{t}-\lambda^{*}\right)^{2}-\frac{s_{t}^{2}}{2 \psi_{i}}
$$

and transition dynamic $\lambda_{t+1}=\lambda_{t}-s_{t}$.

The optimal path corresponding to the linear-quadratic regulator is given by

$$
\begin{gathered}
\lambda_{t+1}=\left[\frac{\mathscr{E}_{i i}+\mathscr{E}_{j j}-2 \mathscr{E}_{i j}+\sqrt{\left(2 \mathscr{E}_{i j}-\mathscr{E}_{i i}-\mathscr{E}_{j j}\right)\left(2 \mathscr{E}_{i j}-\mathscr{E}_{i i}-\mathscr{E}_{j j}+\frac{4}{\psi_{i}}\right)}}{2}\right] \psi_{i} \lambda^{*} \\
+\quad\left[\frac{1}{\psi_{i}}+\frac{2 \mathscr{E}_{i j}-\mathscr{E}_{i i}-\mathscr{E}_{j j}-\sqrt{\left(2 \mathscr{E}_{i j}-\mathscr{E}_{i i}-\mathscr{E}_{j j}\right)\left(2 \mathscr{E}_{i j}-\mathscr{E}_{i i}-\mathscr{E}_{j j}+\frac{4}{\psi_{i}}\right)}}{2}\right] \psi_{i} \lambda_{t},
\end{gathered}
$$


which implies that

$$
s_{t}=\psi_{i}\left[\frac{\mathscr{E}_{i i}+\mathscr{E}_{j j}-2 \mathscr{E}_{i j}+\sqrt{\left(2 \mathscr{E}_{i j}-\mathscr{E}_{i i}-\mathscr{E}_{j j}\right)\left(2 \mathscr{E}_{i j}-\mathscr{E}_{i i}-\mathscr{E}_{j j}+\frac{4}{\rho_{i j}}\right)}}{2}\right]\left(\lambda_{t}-\lambda^{*}\right)
$$

On the other hand, given the optimal tax scheme stated in Proposition 11, the number of $j$-type agents who indoctrinate their children to become $i$-types is given by

$$
\begin{aligned}
\tau_{i t} & =\psi_{i}\left(E_{t}[P(j)]+T_{j t}-E_{t}[P(i)]-T_{i t}\right) \\
& =\psi_{i}\left(\frac{\mathscr{E}_{j j}-\mathscr{E}_{i j}}{2}+\frac{2 \mathscr{E}_{i j}-\mathscr{E}_{i i}-\mathscr{E}_{j j}}{2} \lambda_{t}\right) \\
& =\frac{\psi_{i}}{2}\left(2 \mathscr{E}_{i j}-\mathscr{E}_{i i}-\mathscr{E}_{j j}\right)\left(\lambda_{t}-\lambda^{*}\right) .
\end{aligned}
$$

Equations (6.9) and (6.10) jointly imply the following:

Proposition 12. When $4>3 \psi_{i}\left(2 \mathscr{E}_{i j}-\mathscr{E}_{i i}-\mathscr{E}_{j j}\right), s_{t}>\tau_{i t} ;$ when $4 \leq 3 \psi_{i}\left(2 \mathscr{E}_{i j}-\mathscr{E}_{i i}-\mathscr{E}_{j j}\right)$, $s_{t} \leq \tau_{i t}$.

Proposition 12 implies that when the environment within which socialization takes place is not conducive to cultural change, $\psi_{i}$ is small, either because the cultural distance between the two groups is sufficiently large $\left(\right.$ small $\rho_{i, j}$ ), or the groups are internally very cohesive (large $\rho_{i, i}$ ), then the step sizes on the optimal path chosen by the social planner are larger than those induced by the cultural selection process. The converse is also true. This arrises because there are two "distortions" effecting the agents decisions: First they do not recognize an intertemporal trade-off in that converting their child to the other type today implies a cost to themselves today but saves their type the cost of conversion in the future, this is an intertemporal distortion. Hence the agents tend to convert too little, and this distortion is more important the greater the adjustment cost (the smaller is $\rho_{i, j}$ ) and the smaller is the child's expected payoff benefit from conversion, $2 \mathscr{E}_{i j}-\mathscr{E}_{i i}-\mathscr{E}_{j j}$. The second distortion is the one discussed earlier, in choosing to convert their child to the other type agents do not internalize the cost to other agents in terms their effect on the current probability of an other-type match. This causes them to over convert their children, and this contemporaneous distortion is more important the greater the probability of coordination, $\rho_{i, j}$ and the greater 
the the return to successful coordination, the greater is $2 \mathscr{E}_{i j}-\mathscr{E}_{i i}-\mathscr{E}_{j j}$. The planner of course internalizes both of these effects, so whether $s_{t} \gtrless \tau_{t}$ depends on the relative effects of the intertemporal and contemporaneous distortions on the agents child conversion choices. Examples of policymakers in many countries attempting to force the pace of immigrant or minority assimilation abound and often involve banning or discouraging the use of a language.28

\subsection{A Probabilistic Cultural Transmission Mechanism}

Alternatively, we can think of cultural transmission in a Bisin and Verdier framework (Bisin and Verdier (2001)). Consider the following overlapping generation model. In each generation, an agent engages in economic activities as described in Section 3. At the same time, they become a parent and bear a child, whose cultural type is malleable. The agent is altruistic in regards to the expected earnings of the child and they can exert effort to indoctrinate the child into their own cultural type ${ }^{29}$ The higher effort an agent exerts, the higher probability that the child adopts the agents own cultural type. This process is called direct transmission. In the event that direct transmission fails, the child will pick a role model from society at random and adopt the role model's cultural type. The lifetime utility of a type $i$ agent is now given by

$$
E\left[U_{i} \mid \lambda\right]=E[P(i) \mid \lambda]+E\left[P_{i c} \mid \lambda\right]-\frac{z e_{i}^{2}}{2 \mu_{i}}
$$

which is the sum of their own expected payoff and the expected payoff of their child $\left(E\left[P_{i c} \mid \lambda\right]\right)$ minus a quadratic effort cost $e_{i} \in[0,1]$ where $z$ is a positive constant. The term $\mu_{i}$ again captures the environment in which the $i$-type parent attempts to socialize the child, however

\footnotetext{
${ }^{28}$ Classic examples include the banning of minorities languages such as the Kurdish, Armenian and Greek languages in Turkey. Similarly in Franco's Spain all languages other than Catalan Spanish were banned. In the late 19th and early 20th centuries Hawaiian was banned in all Hawaiian schools.

${ }^{29}$ In Bisin and Verdier (2001), parents are assumed to have "imperfect empathy". That is, instead of being altruistic in regards to the objective expected payoffs of their children, they evaluate the children's consumption bundles according to their own preferences, according to which they are not utility maximizing.
} 
here it is to their own type. We define

$$
\mu_{i}=\left(\rho_{i, i}\right)^{\gamma}\left(\rho_{i, j}\right)^{\delta}
$$

with $\gamma \in(0,1)$ and $\delta \in(-1,1) \cdot 30$ As in the previous section we interpret the effects of $\rho_{i, i}$ and $\rho_{i, j}$ on $\mu_{i}$ as capturing how the local and general environment impact the cost of own-type socialization 31 A larger $\rho_{i, i}$ indicates that the majority type are more culturally cohesive, so family, friends and neighbors are more likely to reinforce a parents attempts to socializer a child making that process less costly. A smaller $\rho_{i, j}$ indicates a greater cultural distance between the two types and represent the more general environment in which an $i$-type parent may try to socialize a child. By letting $\delta \in(-1,1)$ we leave the effect this has on socialization costs ambiguous. Two interpretations suggest themselves; if $\delta>0$ then the socialization cost is decreasing in $\rho_{i, j}$, this suggest that any influences the child may receive from the general environment are not too different from those of the parent and are counteracted relatively inexpensively; if, however, $\delta<0$ then the socialization cost is increasing in $\rho_{i, j}$, here it might be that if the cultural distance between the two groups is large it is easy for a child to distinguish between the socializing influences of the parent and the more general environment, but if the cultural distance is small the child may easily conflate the efforts of the parent with the influences of the general environment making socialization more costly.

Direct transmission succeeds with probability $e_{i}$. If it fails, the child adopts the cultural type of an agent randomly chosen from the population. Hence, the probability that the child becomes an $i$-type agent is written $\mathcal{P}_{i, i}=e_{i}+\left(1-e_{i}\right) \lambda$ and the probability that the child becomes a $j$-type agent equals $\mathcal{P}_{i, j}=\left(1-e_{i}\right)(1-\lambda)$. The expected payoff of the child is given by

$$
\begin{aligned}
E\left[P_{i c} \mid \lambda\right] & =\mathcal{P}_{i, i} E[P(i) \mid \lambda]+\mathcal{P}_{i, j} E[P(j) \mid \lambda] \\
& =\left(e_{i}+\left(1-e_{i}\right) \lambda\right) E[P(i) \mid \lambda]+\left(1-e_{i}\right)(1-\lambda) E[P(j) \mid \lambda]
\end{aligned}
$$

\footnotetext{
${ }^{30}$ Obviously if $\gamma=\delta=0$ we have the same costs as in Bisin and Verdier

${ }^{31}$ Again we omit the obvious similar definition for $\mu_{j}$
} 
Lifetime utility of a type $j$ agent takes a similar form:

$$
E\left[U_{j} \mid \lambda\right]=E[P(j) \mid \lambda]+\delta E\left[P_{j c} \mid \lambda\right]-\frac{z e_{j}^{2}}{2 \mu_{j}},
$$

where $e_{j} \in[0,1]$ is the effort exerted. The expected payoff of the child $E\left[P_{j c}\right]$ is given by

$$
\begin{aligned}
E\left[P_{j c} \mid \lambda\right] & =\mathcal{P}_{j, j} E[P(j) \mid \lambda]+\mathcal{P}_{j, i} E[P(i) \mid \lambda] \\
& =\left(e_{j}+\left(1-e_{j}\right)(1-\lambda)\right) E[P(j) \mid \lambda]+\left(1-e_{j}\right) \lambda E[P(i) \mid \lambda],
\end{aligned}
$$

where $\mathcal{P}_{j, j}=e_{j}+\left(1-e_{j}\right)(1-\lambda)$ is the probability that the child becomes a $j$-type agent and $\mathcal{P}_{j, i}=\left(1-e_{j}\right) \lambda$ is the probability that the child becomes an $i$-type agent.

Assume that $z$ is sufficiently large such that the indoctrination efforts exerted by the agents are bounded away from 1 . The following lemma illustrates the optimal effort exerted by the two types of agents:

Lemma 5. When $E[P(i) \mid \lambda]>E[P(j) \mid \lambda], e_{i}^{*}=\frac{\mu_{i}}{z}(1-\lambda)(E[P(i) \mid \lambda]-E[P(j) \mid \lambda])$ and $e_{j}^{*}=$ 0. When $E[P(i) \mid \lambda]<E[P(j) \mid \lambda], e_{j}^{*}=\frac{\mu_{j}}{z} \lambda(E[P(j) \mid \lambda]-E[P(i) \mid \lambda])$ and $e_{i}^{*}=0$. When $E[P(i) \mid \lambda]=E[P(j) \mid \lambda], e_{i}^{*}=e_{j}^{*}=0$.

By adding time indexes, the dynamic of cultural selection is given by

$$
\begin{aligned}
\lambda_{t+1} & =\lambda_{t}+\left(1-\lambda_{t}\right) \mathcal{P}_{j, i, t}-\lambda_{t} \mathcal{P}_{i, j, t} \\
& =\lambda_{t}+\left(1-\lambda_{t}\right)\left(1-e_{j, t}^{*}\right) \lambda_{t}-\lambda_{t}\left(1-e_{i, t}^{*}\right)\left(1-\lambda_{t}\right),
\end{aligned}
$$

where $\left(1-\lambda_{t}\right)\left(1-e_{j, t}^{*}\right) \lambda_{t}$ is the number of children raised by $j$-type parents eventually become $i$-types (inflow) and $\lambda_{t}\left(1-e_{i, t}^{*}\right)\left(1-\lambda_{t}\right)$ is the number of children raised by $i$-type parents eventually become $j$-types (outflow). 
Given Lemma 4, we rewrite the dynamic of cultural selection as

$$
\begin{aligned}
\lambda_{t+1}= & \lambda_{t}+\lambda_{t}\left(1-\lambda_{t}\right)\left(e_{i, t}^{*}-e_{j, t}^{*}\right) \\
= & \begin{cases}\lambda_{t}-\lambda_{t}^{2}\left(1-\lambda_{t}\right) \frac{\mu_{j}}{z}\left(E_{t}\left[P(j) \mid \lambda_{t}\right]-E_{t}\left[P(i) \mid \lambda_{t}\right]\right) & \text { if } E_{t}\left[P(i) \mid \lambda_{t}\right]<E_{t}\left[P(j) \mid \lambda_{t}\right], \\
\lambda_{t}+\lambda_{t}\left(1-\lambda_{t}\right)^{2} \frac{\mu_{i}}{z}\left(E_{t}\left[P(i) \mid \lambda_{t}\right]-E_{t}\left[P(j) \mid \lambda_{t}\right]\right) & \text { if } E_{t}\left[P(i) \mid \lambda_{t}\right]>E_{t}\left[P(j) \mid \lambda_{t}\right], \\
\lambda_{t} & \text { if } E_{t}\left[P(i) \mid \lambda_{t}\right]=E_{t}\left[P(j) \mid \lambda_{t}\right] .\end{cases}
\end{aligned}
$$

We have the following result:

Proposition 13. $\lambda_{b}$ is asymptotically stable.

Proof: See Appendix.

Proposition 13 demonstrates that the dynamic of cultural selection derived from a probabilistic cultural transmission model based on Bisin and Verdier (2001) is sufficiently smooth such that the interior equilibrium $\lambda_{b}$ is stable, this contrasts with the dynamic of cultural selection derived using an increasing convex cost function.

\section{$7 \quad$ Intermediaries}

In the model above there are two basic frictions. Agents who desire them may fail to obtain other-type matches, and all matched pairs face some chance of a failure to coordinate. Reducing these frictions generates positive expected rents, so it seems reasonable then to expect that specialists of some sort would emerge to perform these function. In this section, we allow for these specialists, termed intermediaries, who can either facilitate matching between the two types of agents or increase the probability of successful coordination in othertype matches ${ }^{32}$ These intermediaries are not directly productive. We ask what the presence

\footnotetext{
${ }^{32}$ When intermediaries facilitate matching this works somewhat like matchmakers discussed by Yavas (1994), examples include the activities of real estate agents and employment agencies. When intermediaries facilitate coordination they are somewhat like the "Middlemen" analyzed by Rubinstein and Wolinsky (1987). In Rubinstein and Wolinsky (1987), intermediaries can be either sellers, buyers or middlemen. Sellers and buyers are in the market infrequently and face search costs as it may take time to match with each
} 
of intermediaries does to the relative proportions of types $i$ and $j$ in the population? This helps us to understand which type of agents are more likely to adopt the role of intermediaries.

\subsection{Intermediaries Facilitate Matching}

Assume that intermediates can help the agents form other-type matches ${ }^{33}$ We consider a case in which, without intermediaries, the two types of agents are segregated from each other. That is agents match with their own type with probability 1 . However, with an intermediary, a $j$-type agent is guaranteed to be matched with an $i$-type agent with probability 1 . This extreme case allows us to gain insights into the problem in a relatively simple way.

Let $\lambda$ denote the fraction of $i$-type agents, $\omega$ denote the fraction of $j$-type agents and $1-\lambda-\omega$ fraction of the agents who adopt the role of intermediaries. We treat intermediaries as the third type of agents denoted by $l$. We initially assume that an intermediary is unconstrained in the number of matches they can facilitate. We again assume that agents can freely choose to be either an $i, j$, or a $l$-type.

Now, for each other-type match, an $i$-type agent, a $j$-type agent and a $l$-type agent bargain over the surplus generated. The threat point of an $i$-type agent is $\frac{\mathscr{E}_{i i}}{2}$. Similarly, the

thread point of a $j$-type agent is $\frac{\mathscr{E}_{j j}}{2}$. The threat point of a $l$-type agent is zero because it is assumed that the intermediary is non-productive elsewhere. This is tantamount to assuming that it is costly to become an intermediary, as agents that become intermediaries give up the outside option of an own-type match. The Nash bargaining problem is given by:

$$
\max _{\theta, \epsilon}\left(\theta \mathscr{E}_{i j}-\frac{\mathscr{E}_{i i}}{2}\right)\left(\epsilon \mathscr{E}_{i j}-\frac{\mathscr{E}_{j j}}{2}\right)\left[(1-\theta-\epsilon) \mathscr{E}_{i j}-0\right]
$$

other, middlemen are always in the market and allow the buyers and seller to reduce search costs. In our analysis when intermediaries improve coordination probabilities these expected gains work like the search cost reductions of Rubinstein and Wolinsky. In a different vein, Li (1998) argues that middlemen exist because they pose private information concerning the consumption goods. Here when intermediaries facilitate othertype matches with probability one they have an informational advantage in matching analagous to the quality information advantage in Li's work.

${ }^{33}$ Bloch and Ryder (2000) develop a model of two sided matching with matchmakers, where the value of a match depends of the quality of the partner. The use of a matchmakers depends on the pricing scheme she adopts for her services. In their model there is no redistribution of the surplus created by a match between the partners. 
By solving this maximization problem, we get

$$
\theta=\frac{\mathscr{E}_{i j}+\mathscr{E}_{i i}-\frac{\mathscr{E}_{i j}}{2}}{3 \mathscr{E}_{i j}} \text { and } \epsilon=\frac{\mathscr{E}_{i j}+\mathscr{E}_{j j}-\frac{\mathscr{E}_{i j}}{2}}{3 \mathscr{E}_{i j}}
$$

Assume as before that $\mathscr{E}_{i i}>\mathscr{E}_{j j}$. Without intermediaries, the whole population of agents become $i$-types. Let us investigate the interior market equilibrium in the presence of intermediaries. In the interior equilibrium, we must have $\lambda>\omega$, because the $i$-types have a higher threat point, and hence will receive higher expected payoff unless they have a lower probability of an other type match than $j$-types. All $j$-type agents receive an other-type match.

Let $\left(\lambda^{*}, \omega^{*}\right)$ denote the interior market equilibrium (if it exists), at which the expected payoffs of three types of agent are given by:

$$
\begin{aligned}
E\left[P(i) \mid \lambda^{*}, \omega^{*}\right] & =\left(\frac{\lambda^{*}-\omega^{*}}{\lambda^{*}}\right) \frac{\mathscr{E}_{i i}}{2}+\left(\frac{\omega^{*}}{\lambda^{*}}\right) \theta \mathscr{E}_{i j} \\
E\left[P(j) \mid \lambda^{*}, \omega^{*}\right] & =\epsilon \mathscr{E}_{i j} ; \\
E\left[P(l) \mid \lambda^{*}, \omega^{*}\right] & =\left(\frac{\omega^{*}}{1-\lambda^{*}-\omega^{*}}\right)(1-\theta-\epsilon) \mathscr{E}_{i j}
\end{aligned}
$$

Here we assume that an intermediary can handle arbitrary number of matches. Hence, an intermediary's expected payoff is equal to the rent extracted from each bargain times the number of matches handled $\frac{\omega^{*}}{1-\lambda^{*}-\omega^{*}}$.

In a stable interior equilibrium, we must have $E\left[P(i) \mid \lambda^{*}, \omega^{*}\right]=E\left[P(j) \mid \lambda^{*}, \omega^{*}\right]=E\left[P(l) \mid \lambda^{*}, \omega^{*}\right]$. Moreover, the following three conditions must be satisfied:

$$
\epsilon \mathscr{E}_{i j}>\frac{\mathscr{E}_{i i}}{2}, \omega \in(0,1), \lambda>\omega
$$

The first condition indicates that the rent extracted by a $j$-type agent must be larger than the payoff an $i$-type agent can obtain from an own-type match, otherwise all agents would choose to be $i$-types. We find that when $\mathscr{E}_{i j}$ is sufficiently high, the above three conditions are satisfied. In other words, when an other-type match is sufficiently productive, an equilibrium in which intermediaries emerge is the only stable prediction.

However, in reality, each intermediary may only be able to handle a limited number of 
matches due to ability and/or time constraints. Let $v$ denote the number of matches each intermediary can facilitate. We are interested in investigating whether the socially optimal proportion of intermediaries differs from the market equilibrium given that each intermediary is constrained to facilitate at most $v$ matches. There are two possible cases: 1) $v<\frac{\omega^{*}}{1-\lambda^{*}-\omega^{*}}$; 2) $v>\frac{\omega^{*}}{1-\lambda^{*}-\omega^{*}}$.

In the first case the constraint on the intermediaries is binding, $v<\frac{\omega^{*}}{1-\lambda^{*}-\omega^{*}}$, and the market interior equilibrium $\left(\lambda^{*}, \omega^{*}\right)$ without constraints cannot be obtained. Instead there are two potential market equilibria: 1) an interior equilibrium in which not all $j$-type agents are matched with $i$-type agents (contrary to $\left.\left(\lambda^{*}, \omega^{*}\right)\right)$; or, 2) a corner equilibrium in which the whole population of agents choose to be $i$-types. This equilibrium occurs when $v$ is sufficiently small such that the intermediaries' expected payoff are so low that no agent would choose to be an l-type.

Let $(\tilde{\lambda}, \tilde{\omega})$ denote the interior equilibrium with constraints (if it exists) and let $\tilde{x}=$ $v(1-\tilde{\lambda}-\tilde{\omega})$ be the number of other-type matches. The expected payoffs of the agents are given by

$$
\begin{aligned}
E[P(i) \mid \tilde{\lambda}, \tilde{\omega}] & =\left(\frac{\tilde{\lambda}-\tilde{x}}{\tilde{\lambda}}\right) \frac{\mathscr{E}_{i i}}{2}+\left(\frac{\tilde{x}}{\tilde{\lambda}}\right) \theta \mathscr{E}_{i j} \\
E[P(j) \mid \tilde{\lambda}, \tilde{\omega}] & =\left(\frac{\tilde{\omega}-\tilde{x}}{\tilde{\omega}}\right) \frac{\mathscr{E}_{j j}}{2}+\left(\frac{\tilde{x}}{\tilde{\omega}}\right) \epsilon \mathscr{E}_{i j} ; \\
E[P(l) \mid \tilde{\lambda}, \tilde{\omega}] & =\tilde{x}(1-\theta-\epsilon) \mathscr{E}_{i j} .
\end{aligned}
$$

We must have $\tilde{x}<\tilde{\omega}<\tilde{\lambda}$. $\tilde{x}<\tilde{\omega}$ because in this interior equilibrium not all $j$-type agents are matched with $i$-type agents. $\tilde{\omega}<\tilde{\lambda}$ is necessary for the expected payoff of an $i$-type agent to equal that of an $j$-type agent.

Now let us look at the utilitarian social planner's problem. Let $x$ denote the total number of matches that intermediaries facilitate. Then total welfare equals

$$
E[W \mid \lambda, \omega]=(\lambda-x) \frac{\mathscr{E}_{i i}}{2}+(\omega-x) \frac{\mathscr{E}_{j j}}{2}+x \mathscr{E}_{i j}
$$

with constraints $x \leq \omega \leq \lambda$ and $x \leq v(1-\lambda-\omega)$.

Let $\lambda_{S P}$ denote the optimal number of $i$-type agents, $\omega_{S P}$ denote the optimal number 
of $j$-type agents and $x_{S P}$ denote the optimal number of matches facilitated by each $l$-type agent. We have the following result:

Proposition 14. When $\mathscr{E}_{i j}>\mathscr{E}_{i i}+\frac{\mathscr{E}_{j j}}{2}, \lambda_{S P}=\omega_{S P}=x_{S P}=\frac{v}{1+2 v}$. When $\mathscr{E}_{i j}<\mathscr{E}_{i i}+\frac{\mathscr{E}_{j j}}{2}$, $\lambda_{S P}=1, \omega_{S P}=x_{S P}=0$.

Proof: Total welfare can be rewritten as

$$
E[W \mid \lambda, \omega]=\lambda\left(\frac{\mathscr{E}_{i i}}{2}\right)+\omega\left(\frac{\mathscr{E}_{j j}}{2}\right)+x\left(\mathscr{E}_{i j}-\frac{\mathscr{E}_{i i}}{2}-\frac{\mathscr{E}_{j j}}{2}\right)
$$

When $\mathscr{E}_{i j}-\frac{\mathscr{E}_{i i}}{2}-\frac{\mathscr{E}_{j j}}{2}>\frac{\mathscr{E}_{i i}}{2}$, i.e., $\mathscr{E}_{i j}>\mathscr{E}_{i i}+\frac{\mathscr{E}_{j j}}{2}$, the social planner wishes to maximize $x$ which occurs where the two constraints $x \leq \omega \leq \lambda$ and $x \leq v(1-\lambda-\omega)$ are binding. This gives us $\lambda_{S P}=\omega_{S P}=x_{S P}=\frac{v}{1+2 v}$. When $\mathscr{E}_{i j}<\mathscr{E}_{i i}+\frac{\mathscr{E}_{j j}}{2}$, the social planner wishes to maximize $\lambda$, which leads to $\lambda_{S P}=1, \omega_{S P}=x_{S P}=0$. Q.E.D.

We have the following immediate result:

Corollary 4. If $\mathscr{E}_{i j}>\mathscr{E}_{i i}+\frac{\mathscr{E}_{j j}}{2}$, then $x_{S P}>\tilde{x}>0$.

Proof: The market interior equilibrium satisfies that $\tilde{x}=v(1-\tilde{\lambda}-\tilde{\omega}), \tilde{x}<\tilde{\omega}<\tilde{\lambda}$, which implies that $\tilde{x}<v(1-2 \tilde{x})$. Hence, we have $\tilde{x}<\frac{v}{1+2 v}=x_{S P}$. Q.E.D.

Proposition 14 and Corollary 4 show that when $\mathscr{E}_{i j}>\mathscr{E}_{i i}+\frac{\mathscr{E}_{j j}}{2}$, i.e., when other-type matching is sufficiently productive, the social planner always wants more intermediaries than the market equilibrium. Otherwise, the social planner prefers to induce a homogeneous $i$-type society. The intuition here is that when an individual agent chooses whether or not to be an intermediary they only consider their own benefits, a planner however also internalizes the benefits to other agents that arise from the increased probability of an other-type match.

Next, we consider the second case in which $v>\frac{\omega^{*}}{1-\lambda^{*}-\omega^{*}}$. In this case, the market interior equilibrium $\left(\lambda^{*}, \omega^{*}\right)$ can be obtained. The social planner's problem is identical to that in the previous case. Hence, Proposition 14 still holds. When $\mathscr{E}_{i j}>\mathscr{E}_{i i}+\frac{\mathscr{E}_{j j}}{2}$, in the social optimum, the proportion of intermediaries equals $\frac{x_{S P}}{v}=\frac{1}{2 v+1}$, which monotonically decreases in $v$. In other words, as the number of matches each intermediary can facilitate increases, the social 
planner desires fewer intermediaries. Moreover, there exists a $\underline{v}$, such that for any $v>\underline{v}$, $\frac{1}{2 v+1}<1-\lambda^{*}-\omega^{*}$. That is, the social planner prefers fewer intermediaries than the market equilibrium.

\subsection{Intermediaries Facilitate Coordination}

Suppose now the intermediaries are able to increase the probability of successful coordination in the other-type matches. How much the intermediaries can improve on the coordination probability depends on the bundle of characteristics that define a cultural type, and which aspects of culture are the most relevant for coordination purposes. If the characteristics of the cultural most relevant to facilitating coordination are elements such as languages or customs, let us call these "social traits", then presumably the intermediaries cannot understand either culture better than they can understand themselves. In this case they can at best raise $\rho_{i, j} \rightarrow \min \left\{\rho_{i, i}, \rho_{j, j}\right\}$.

On the other hand, if the characteristics of culture most relevant to coordination involve agents' culturally influenced "personal traits" such as diplomatic skills or the ability to empathize, then $\rho_{i i}$ and $\rho_{j j}$ represent how agents' personal traits affect their own-cultural coordination respectively. Hence, intermediaries from group $i$ can raise $\rho_{i j}$ to $\rho_{i i}$, while intermediaries from group $j$ can raise $\rho_{i j}$ to $\rho_{j j}$.

Finally it might be that the ability to improve coordination depends on an intermediaries "cultural experience", where the type that has more experience of other-type matching is better able to increase the probability of coordination. We capture this idea by supposing that if $\rho_{i, j}^{i}$ as the probability of successful coordination if the intermediary is drawn from the population of $i$-types. Then their probability of facilitating coordination might be defined by $\rho_{i, j}^{i}=\min \left\{\rho_{i, i}, \rho_{j, j}\right\}(1-\lambda)$. A Similar expression holds if an intermediary is drawn from the population of for $j$-types.

\subsubsection{Coordination Based on Social Traits}

We consider first the the case where the ability to facilitate coordination depends on social traits such as languages or customs. In this case, intermediaries can raise $\rho_{i, j} \rightarrow \min \left\{\rho_{i, i}, \rho_{j, j}\right\}$ 
regardless of which group they originated from. Let $\lambda$ denote the fraction of $i$-type agents, $\omega$ denote the fraction of $j$-type agents and $1-\lambda-\omega$ denote the fraction of the agents who adopt the role of intermediaries. We treat intermediaries as a third type of agents denote by $l$.

For each other-type match that involves the services of an intermediary, an $i$-type agent, a $j$-type agent and a $l$-type agent bargain over the surplus generated by the match. The threat point of an $i$-type agent is $\beta^{*} \mathscr{E}_{i j}$, which is the expected payoff that an $i$-type agent can obtain in a other-type match without the help of an intermediary. Similarly, the threat point of a $j$-type agent is $\left(1-\beta^{*}\right) \mathscr{E}_{i j}$. The threat point of a $l$-type agent is zero because if the three parties cannot come to an agreement, then the intermediary is again assumed to be unproductive elsewhere ${ }^{34}$ The Nash bargaining problem is as follows:

$$
\begin{aligned}
\max _{\theta, \epsilon} & \left(\theta \min \left\{\rho_{i, i}, \rho_{j, j}\right\} R_{i j}-\beta^{*} \mathscr{E}_{i j}\right)\left(\epsilon \min \left\{\rho_{i, i}, \rho_{j, j}\right\} R_{i j}-\left(1-\beta^{*}\right) \mathscr{E}_{i j}\right) \\
& \times\left((1-\theta-\epsilon) \min \left\{\rho_{i, i}, \rho_{j, j}\right\} R_{i j}-0\right) .
\end{aligned}
$$

By solving this maximization problem, we get

$$
\begin{aligned}
\theta & =\frac{\min \left\{\rho_{i, i}, \rho_{j, j}\right\} R_{i j}+2 \beta^{*} \mathscr{E}_{i j}-\left(1-\beta^{*}\right) \mathscr{E}_{i j}}{3 \min \left\{\rho_{i, i}, \rho_{j, j}\right\} R_{i j}} \\
\epsilon & =\frac{\min \left\{\rho_{i, i}, \rho_{j, j}\right\} R_{i j}+2\left(1-\beta^{*}\right) \mathscr{E}_{i j}-\beta^{*} \mathscr{E}_{i j}}{3 \min \left\{\rho_{i, i}, \rho_{j, j}\right\} R_{i j}}
\end{aligned}
$$

In the interior equilibrium, the expected payoff of three types of agents are given by:

$$
\begin{aligned}
E[P(i)] & =\left(\frac{\lambda}{\lambda+\omega}\right) \frac{\mathscr{E}_{i i}}{2}+\left(\frac{\omega}{\lambda+\omega}\right) \theta \min \left\{\rho_{i, i}, \rho_{j, j}\right\} R_{i j} \\
E[P(j)] & =\left(\frac{\omega}{\lambda+\omega}\right) \frac{\mathscr{E}_{j j}}{2}+\left(\frac{\lambda}{\lambda+\omega}\right) \epsilon \min \left\{\rho_{i, i}, \rho_{j, j}\right\} R_{i j} \\
E[P(l)] & =\left(\frac{\lambda \omega}{1-\lambda-\omega}\right)(1-\theta-\epsilon) \min \left\{\rho_{i, i}, \rho_{j, j}\right\} R_{i j} .
\end{aligned}
$$

Similar to the previous subsection, an intermediary's expected payoff is equal to the

\footnotetext{
${ }^{34} \mathrm{We}$ are assuming implicitly here that if negotiations break down the $i$ and $j$ type players recognize that they have achieved a beneficial other-type match and will maintain this match and bargain directly with each other. They do not revert to the "outside-option" of an own-type match.
} 
total rent that all the intermediaries extract from bargaining divided by the proportion of intermediaries.

Let $\kappa=\frac{\omega}{\lambda}$ denote the ratio between the proportion of $j$-type agents and that of $i$-type agents. By equating $E[P(i)]=E[P(j)]$, we have

$$
\kappa=\frac{\frac{4}{3}\left(\min \left\{\rho_{i, i}, \rho_{j, j}\right\} R_{i j}-\mathscr{E}_{i j}\right)+2 \mathscr{E}_{i j}+\mathscr{E}_{j j}-3 \mathscr{E}_{i i}}{\frac{4}{3}\left(\min \left\{\rho_{i, i}, \rho_{j, j}\right\} R_{i j}-\mathscr{E}_{i j}\right)+2 \mathscr{E}_{i j}+\mathscr{E}_{i i}-3 \mathscr{E}_{j j}}>\frac{2 \mathscr{E}_{i j}+\mathscr{E}_{j j}-3 \mathscr{E}_{i i}}{2 \mathscr{E}_{i j}+\mathscr{E}_{i i}-3 \mathscr{E}_{j j}}=\frac{1-\lambda_{b}}{\lambda_{b}}
$$

Hence, we conclude that in the case where the relevant element of culture for facilitating coordination is a social trait such as language or custom, intermediaries mostly come from the majority group ( $i$-type agents). This follows from our earlier comparative statics results which stated that an increase in the expected payoff from an other-type match disproportionately benefited the minority group. This was because they were more likely to achieve such a match than the majority. Here the same is true, so an increase in the expected value of other-type matches due to the emergence of intermediaries again disproportionately benefits the minority $j$-types, hence relatively fewer intermediaries are drawn from their population.

An interesting example of this type of intermediation might be the role of Pocahontas and other members of the Powhatan tribe who facilitated trade between the majority local American tribes and the minority English colonists in early 17th century Jamestown. Several Powhatans lived amongst the English and learned their language and about their culture, but refused to become acultured, resisting, for example, having their children attend English schools.

\subsubsection{Coordination Based on Personal Traits}

Next, we discuss the case that cultural types represent personal traits ${ }^{35}$ In this case, the intermediaries from the two groups are asymmetric because they can increase the probability

\footnotetext{
${ }^{35}$ In an article "Ideal Qualities of a Successful Diplomat" Robert Blackwill of the Belfer Center for Science and International Affairs at the John F. Kennedy School of Government, lists several personal characteristics that make a good diplomat but may be a product of the individuals culture. These include the tendency to analyze before prescribe, to be verbally fluent and concise, to be highly attentive to detail, and to be willing to accept risk. All traits that might be learned from an individuals original culture. These views are shared by Karl Gruber, ex Austrian Foreign Minister and Austrian Ambassador to the United States, Spain, Switzerland, and West Germany in an article in eDiplomat (2016). Gruber adds the ability to empathize to Blackwill's list.
} 
of successful coordination to different levels. In the previous case, the agents in a other-type match can either hire an intermediary or not. Now, the agents have the options to choose from the two different types of intermediaries or not to hire one. Hence, those intermediaries from the group that has a lower ability to facilitate other-type coordination would be crowded out.

We are interested in the scenario in which $\mathscr{E}_{i i}>\mathscr{E}_{j j}$ and $\rho_{i i}<\rho_{j j}$. In this scenario, without intermediaries, $i$-type agents are the majority in the population. However, $j$-type agents are more suitable to be intermediaries because their personal traits facilitate coordination. Therefore, all the intermediaries come from the $j$ group in the interior equilibrium.

As in the previous section $\lambda$ denotes the fraction of $i$-type agents, $\omega$ denotes the fraction of $j$-type agents and $1-\lambda-\omega$ the fraction of the agents who adopt the role of intermediaries who are again denoted by $l$. Recall that here all intermediaries must come from group $j$.

The Nash bargaining problem is given as follows:

$$
\max _{\theta, \epsilon}\left(\theta \rho_{j j} R_{i j}-\beta^{*} \mathscr{E}_{i j}\right)\left(\epsilon \rho_{j j} R_{i j}-\left(1-\beta^{*}\right) \mathscr{E}_{i j}\right)\left((1-\theta-\epsilon) \rho_{j j} R_{i j}-0\right)
$$

By solving this maximization problem, we get

$$
\theta=\frac{\rho_{j j} R_{i j}+2 \beta^{*} \mathscr{E}_{i j}-\left(1-\beta^{*}\right) \mathscr{E}_{i j}}{3 \rho_{j j} R_{i j}} \text { and } \epsilon=\frac{\rho_{j j} R_{i j}+2\left(1-\beta^{*}\right) \mathscr{E}_{i j}-\beta^{*} \mathscr{E}_{i j}}{3 \rho_{j j} R_{i j}}
$$

In the interior equilibrium, the expected payoff of three types of agents are given by:

$$
\begin{aligned}
E[P(i)] & =\left(\frac{\lambda}{\lambda+\omega}\right) \frac{\mathscr{E}_{i i}}{2}+\left(\frac{\omega}{\lambda+\omega}\right) \theta \rho_{j j} R_{i j} \\
E[P(j)] & =\left(\frac{\omega}{\lambda+\omega}\right) \frac{\mathscr{E}_{j j}}{2}+\left(\frac{\lambda}{\lambda+\omega}\right) \epsilon \rho_{j j} R_{i j} \\
E[P(l)] & =\left(\frac{\lambda \omega}{1-\lambda-\omega}\right)(1-\theta-\epsilon) \rho_{j j} R_{i j} .
\end{aligned}
$$

Since the intermediaries all come from group $j$, the ratio between the proportion of $j$-type agents and the proportion of $i$-type agents is measured by $\frac{1-\lambda}{\lambda}$ and we have:

$$
\frac{1-\lambda}{\lambda}>\frac{\omega}{\lambda}=\frac{\frac{4}{3}\left(\rho_{j j} R_{i j}-\mathscr{E}_{i j}\right)+2 \mathscr{E}_{i j}+\mathscr{E}_{j j}-3 \mathscr{E}_{i i}}{\frac{4}{3}\left(\rho_{j j} R_{i j}-\mathscr{E}_{i j}\right)+2 \mathscr{E}_{i j}+\mathscr{E}_{i i}-3 \mathscr{E}_{j j}}>\frac{1-\lambda_{b}}{\lambda_{b}}
$$


Hence, the minority group $j$ expands due to the possibility of becoming intermediaries. The intuition here is that intermediaries create extra rents so some $j$-types choose to be intermediaries. Ceteris paribus this reduces the number of $j$-types involved in matching, making cross-cultural matches more likely for the $j$-types and less likely for $i$-types. The expected value of being a $j$-type relative to an $i$-type rises inducing more agents to choose to choose to become $j$-types.

\subsubsection{Coordination Based on Cultural Experience}

The idea here is that the general "cultural experience" of meeting other types matters in the role of intermediaries. If this is the case and we write $\rho_{i, j}^{i}$ as the probability of successful coordination if the intermediary is an $i$-type. Then we might define

$$
\rho_{i, j}^{i}=\min \left\{\rho_{i, i}, \rho_{j, j}\right\}(1-\lambda) \text { and } \rho_{i, j}^{j}=\min \left\{\rho_{i, i}, \rho_{j, j}\right\} \lambda
$$

This tells us that, away from the matching/coordination game we model, the minority $j$ types meet majority $i$-types with greater frequency than the converse, they therefore have more cross-cultural experience and are better intermediaries ${ }^{36}$

When $\lambda>\frac{1}{2}$, we have $\rho_{i, j}^{i}<\rho_{i, j}^{j}$. In this case, intermediaries only come from the $j$ group. On the other hand, When $\lambda>\frac{1}{2}$, we have $\rho_{i, j}^{i}<\rho_{i, j}^{j}$. In this case, intermediaries only come from the $i$ group. We have the following result:

Proposition 15. If an interior equilibrium $\lambda$ exists, then $\lambda \geq \frac{1}{2}$.

Proof: See Appendix.

Proposition 15 states that if an interior equilibrium exists, $j$-type agents must be the minority and intermediaries should all come from group $j$. The intuition is that if instead $i$-type agents are the minority, they not only obtain a higher share of the rents in crosscultural matches, but they also have a higher chance to engage in cross-cultural matches. Therefore, the $i$ group must expand until it becomes the majority.

Finally, let us briefly discuss what the optimal number of intermediaries would be chosen

\footnotetext{
${ }^{36}$ This is what sociologists term "acculturation without assimilation".
} 
by a social planner when intermediaries facilitate coordination. Assume that each trader can facilitate coordination for a limited number $(\nu)$ of cross-cultural matches. Similar to the intuitions we derive in Section 7.1, when $\nu$ is sufficiently small, the social planner want more intermediaries than the market equilibrium; when $\nu$ is sufficiently large, the social planner want fewer intermediaries than the market equilibrium.

\section{Conclusion/Extensions}

The analysis presented above tackles the currently pressing question of what might be expected to happen to societies comprised of more than one distinct cultural group? Will there be assimilation of one group by another or will they remain distinct? Will the cultural dynamics lead to an efficient outcome or not? And to each of these questions we can add "why?" We have addressed these questions by developing a theoretical model of the transmission of culture based on novel primitives. We assume that there are skill complementarities between the two cultural types but also communication problems. Allowing agents from the two cultural groups to be matched at random to play a coordination game, and then letting the players bargain over the fruits of successful coordination allows us to gain many insights into the questions posed. We are able to explain when and if one cultural group assimilates another as a function of the within and across group probabilities of successful coordination and the relative productivities of own-type and other-type matches. Generally we find that when coexistence of the two cultural types is desirable from a welfare perspective then the market solution involves the minority group being too small. This provides an explanation for many countries adopting policies that promote multiculturalism. How-

ever, in a dynamic context our analysis points to the possibility that agents in pursuit of economic advantages will overreact to such an extent in terms of cross-cultural transmission that policies to encourage assimilation might be desirable. When the market equilibrium in our model is interior we show that more interaction between cultural groups is always desirable, indeed in our specific context it is Pareto improving.

In an extension to our analysis we showed that it is natural for agents to appear that act as intermediaries between the two cultural groups. These intermediaries may facilitate 
improved matching or coordination or both. We are able to identify the conditions under which the intermediaries are drawn from the minority or majority cultural group and whether their numbers are efficient. Again we obtain our results from the probabilities successful own-type and other-type coordination, interpreted as before as measures of within group cohesiveness and the cultural distance between groups.

There are several potential extensions to our analysis. If we were to reinterpret and model more carefully the outside options of the cultural types, we might gain some insight into the distribution of black market activities across types. Also, it might be possible to allow cultural choice to be continuous so as to explore whether equilibria exist with multiple types even when agents could in principle choose a form of "melting pot" where a common mix of cultural types emerges. These are of course questions for future study.

\section{Appendix}

\section{Proof of Corollary 3}

$E_{1}\left[P(i), n, \lambda^{*}(n)\right]=E_{1}\left[P(j), n, \lambda^{*}(n)\right]$, for any $n>\underline{n}$. Moreover, (5.9) and (5.10) imply that

$$
\begin{aligned}
E_{1}\left[P(i), n+1, \lambda^{*}(n)\right] & -E_{1}\left[P(i), n, \lambda^{*}(n)\right] \\
= & \left(\mathscr{E}_{j, i}-\frac{\mathscr{E}_{i, i}}{2}-\frac{\mathscr{E}_{j, j}}{2}\right)\left(\frac{1-\lambda_{n+1}}{2}-\sum_{t=1}^{n} \frac{1-\lambda_{t}}{2^{n+2-t}}\right) ; \\
E_{1}\left[P(j), n+1, \lambda^{*}(n)\right] & -E_{1}\left[P(j), n, \lambda^{*}(n)\right] \\
= & \left(\mathscr{E}_{j, i}-\frac{\mathscr{E}_{i, i}}{2}-\frac{\mathscr{E}_{j, j}}{2}\right)\left(\frac{\lambda_{n+1}}{2}-\sum_{t=1}^{n} \frac{\lambda_{t}}{2^{n+2-t}}\right) .
\end{aligned}
$$

This in turn implies that

$$
\begin{aligned}
& E_{1}\left[P(j), n+1, \lambda^{*}(n)\right]-E_{1}\left[P(i), n+1, \lambda^{*}(n)\right] \\
= & \left(E_{1}\left[P(j), n+1, \lambda^{*}(n)\right]-E_{1}\left[P(j), n, \lambda^{*}(n)\right]\right) \\
& -\left(E_{1}\left[P(i), n+1, \lambda^{*}(n)\right]-E_{1}\left[P(i), n, \lambda^{*}(n)\right]\right. \\
= & \left(\mathscr{E}_{j, i}-\frac{\mathscr{E}_{i, i}}{2}-\frac{\mathscr{E}_{j, j}}{2}\right)\left(\frac{2 \lambda_{n+1}-1}{2}-\sum_{t=1}^{n} \frac{2 \lambda_{t}-1}{2^{n+2-t}}\right)
\end{aligned}
$$


Since $\lambda^{*}(n)>\frac{1}{2}$, we know $\lambda_{t}$ monotonically increases in $t$. Hence, we have $\frac{2 \lambda_{n+1}-1}{2}-$ $\sum_{t=1}^{n} \frac{2 \lambda_{t}-1}{2^{n+2-t}}>\frac{2 \lambda_{n+1}-1}{2}-\sum_{t=1}^{n} \frac{2 \lambda_{n+1}-1}{2^{n+2-t}}=\frac{2 \lambda_{n+1}-1}{2^{n+1}}>0$. This indicates that $E_{1}[P(j), n+$ $\left.1, \lambda^{*}(n)\right]>E_{1}\left[P(i), n+1, \lambda^{*}(n)\right]$.

Given that $E_{1}[P(i), t, \lambda]$ is strictly decreasing in $\lambda$ and $E_{1}[P(j), t, \lambda]$ is strictly increasing in $\lambda$, for any $t>0$. We conclude that $\lambda^{*}(n+1)<\lambda^{*}(n)$. Q.E.D.

\section{Proof of Proposition 11}

By imposing the tax scheme described by $T_{i t}$ and $T_{j t}$, it is straightforward to show that $\lambda^{*}$ is the unique interior equilibrium. By the same reasoning as in the proof of Proposition 10, we can safely ignore the population bounds (0 and 1) and rewrite the proportion of the population of type $i$ after $t$ periods given an initial state $\lambda_{0} \in[0,1]$ as

$$
\lambda_{t+1}= \begin{cases}\left(1+\psi_{i}\left(\frac{\mathscr{E}_{i, i}+\mathscr{E}_{j, j}}{2}-\mathscr{E}_{i, j}\right)\right) \lambda_{t}+\psi_{i} \frac{\mathscr{E}_{j, j}-\mathscr{E}_{i, j}}{2} & \text { if } \lambda_{t}>\lambda_{b} \\ \left(1+\psi_{j}\left(\frac{\mathscr{E}_{i, i}+\mathscr{E}_{j, j}}{2}-\mathscr{E}_{i, j}\right)\right) \lambda_{t}+\psi_{j} \frac{\mathscr{E}_{j, j}-\mathscr{E}_{i, j}}{2} & \text { if } \lambda_{t}<\lambda_{b} \\ \lambda_{t} & \text { if } \lambda_{t}=\lambda_{b}\end{cases}
$$

If both $\left|1+\psi_{i}\left(\frac{\mathscr{E}_{i, i}+\mathscr{E}_{j, j}}{2}-\mathscr{E}_{i, j}\right)\right|<1$ and $\left|1+\psi_{j}\left(\frac{\mathscr{E}_{i, i}+\mathscr{E}_{j, j}}{2}-\mathscr{E}_{i, j}\right)\right|<1$, then $\lambda_{b}$ is globally asymptotically stable. If either $\left|1+\psi_{i}\left(\frac{\mathscr{E}_{i, i}+\mathscr{E}_{j, j}}{2}-\mathscr{E}_{i, j}\right)\right|>1$ or $\left|1+\psi_{j}\left(\frac{\mathscr{E}_{i, i}+\mathscr{E}_{j, j}}{2}-\mathscr{E}_{i, j}\right)\right|>1$, then $\lambda_{b}$ is repelling. Q.E.D.

\section{Proof of Proposition 13}

Rewrite the dynamic as $\lambda_{t+1}=f\left(\lambda_{t}\right)$, we have $\lim _{\lambda_{t} \rightarrow \lambda_{b}}^{-} f^{\prime}\left(\lambda_{t}\right)=1-\frac{\mu_{j}}{z} B(1+B / A)^{2}$ and $\lim _{\lambda_{t} \rightarrow \lambda_{b}}^{+} f^{\prime}\left(\lambda_{t}\right)=1+\frac{\mu_{i}}{z} B^{2} / A^{2}(A+B)$, where $A=\left(\frac{\mathscr{E}_{i, i}+\mathscr{E}_{j, j}}{2}-\mathscr{E}_{i, j}\right)<0$ and $B=$ $\left(\frac{\mathscr{E}_{i, i}-3 \mathscr{E}_{j, j}}{4}+\frac{\mathscr{E}_{i, j}}{2}\right)>0 . e_{i}^{*}<1$ implies that $\frac{\mu_{i}}{z} B<1$, where the LHS is an i parent's effort when $\lambda=1 . e_{j}^{*}<1$ implies that $-\frac{\mu_{j}}{z}(A+B)<1$, where LHS is an j parent's effort when $\lambda=0$. One can also show that $\frac{(A+B) B}{A^{2}}>-2$. These together imply that $1-\frac{\mu_{j}}{z} B(1+B / A)^{2} \in(-1,1)$ and $1+\frac{\mu_{i}}{z} B^{2} / A^{2}(A+B) \in(-1,1)$. Hence, $\lambda_{b}$ is asymptotically stable. Q.E.D. 


\section{Proof of Proposition 15}

We prove it by contradiction. Suppose that $\lambda<\frac{1}{2}$, then we have $\rho_{i, j}^{i}<\rho_{i, j}^{j}$ and intermediaries only come from the $i$ group. Let $\nu$ denote the fraction of $i$-type agents, $1-\lambda$ denote the fraction of $j$-type agents and $\lambda-\nu$ fraction of the agents who adopt the role of intermediaries.

The Nash bargaining problem is given as follows:

$$
\max _{\theta^{\prime}, \epsilon^{\prime}}\left(\theta^{\prime} \rho_{i j}^{i} R_{i j}-\beta^{*} \mathscr{E}_{i j}\right)\left(\epsilon^{\prime} \rho_{i j}^{i} R_{i j}-\left(1-\beta^{*}\right) \mathscr{E}_{i j}\right)\left(\left(1-\theta^{\prime}-\epsilon^{\prime}\right) \rho_{i j}^{i} R_{i j}-0\right)
$$

By solving this maximization problem, we get

$$
\theta^{\prime}=\frac{\rho_{i j}^{i} R_{i j}+2 \beta^{*} \mathscr{E}_{i j}-\left(1-\beta^{*}\right) \mathscr{E}_{i j}}{3 \rho_{i j}^{i} R_{i j}} \text { and } \epsilon^{\prime}=\frac{\rho_{i j}^{i} R_{i j}+2\left(1-\beta^{*}\right) \mathscr{E}_{i j}-\beta^{*} \mathscr{E}_{i j}}{3 \rho_{i j}^{i} R_{i j}}
$$

In the interior equilibrium, the expected payoff of three types of agents are given by:

$$
\begin{aligned}
& E[P(i)]=\left(\frac{\nu}{\nu+1-\lambda}\right) \frac{\mathscr{E}_{i i}}{2}+\left(\frac{1-\lambda}{\nu+1-\lambda}\right) \theta^{\prime} \rho_{i j}^{i} R_{i j} \\
& E[P(j)]=\left(\frac{1-\lambda}{\nu+1-\lambda}\right) \frac{\mathscr{E}_{j j}}{2}+\left(\frac{\nu}{\nu+1-\lambda}\right) \epsilon^{\prime} \rho_{i j}^{i} R_{i j} \\
& E[P(l)]=\left(\frac{\nu(1-\lambda)}{\lambda-\mu}\right)\left(1-\theta^{\prime}-\epsilon^{\prime}\right) \rho_{i j}^{i} R_{i j} .
\end{aligned}
$$

By equating $E[P(i)]=E[P(j)]$, we have

$$
\frac{1-\lambda}{\lambda}<\frac{1-\lambda}{\nu}=\frac{\frac{4}{3}\left(\rho_{i j}^{i} R_{i j}-\mathscr{E}_{i j}\right)+2 \mathscr{E}_{i j}+\mathscr{E}_{j j}-3 \mathscr{E}_{i i}}{\frac{4}{3}\left(\rho_{i j}^{i} R_{i j}-\mathscr{E}_{i j}\right)+2 \mathscr{E}_{i j}+\mathscr{E}_{i i}-3 \mathscr{E}_{j j}}
$$

The LHS of inequality 8.10 is decreasing in $\lambda$ and is always larger than 1 when $\lambda<\frac{1}{2}$. The RHS of 8.10 inequality is also decreasing in $\lambda$ and its maximum value is less than 1 (its maximum value is attained when $\lambda=0$ ). Therefore, inequality 8.10 is impossible to hold.

This indicates that if there exists an interior equilibrium $\lambda$, it cannot be less than $\frac{1}{2}$. Q.E.D. 


\section{References}

[1] Ahern, Kenneth R., Daniele Daminelli, and Cesare Fracassi (2015): "Lost in Translation? The Effect of Cultural Values on Mergers around the World." Journal of Financial Economics 117(1): 165-189.

[2] Alesina, Alberto, Enrico Spolaore and Romain Wacziarg (2000): "Economic Integration and Political Disintegration." American Economic Review, 90(5): 1276-1296.

[3] Berliant, Marcus, and Masahisa Fujita (2008): "Knowledge Creation as a Square Dance on the Hilbert Cube." International Economic Review 49(4): 1251-1295.

[4] Bertsekas, Dimitri P., and Dimitri P. Bertsekas (1995): Dynamic Programming and Optimal Control. Vol. 1(2). Belmont, MA: Athena Scientific.

[5] Bisin, Alberto, and Thierry Verdier (2000): "Beyond the Melting Port: Cultural Transmission, Marriage and the Evolution of Ethnic and Religious Traits." The Quarterly Journal of Economics 115(1): 955-988.

[6] Bisin, Alberto, and Thierry Verdier (2001): "The Economics of Cultural Transmission and the Dynamics of Preferences." Journal of Economic Theory 97(2): 298-319.

[7] Bisin, Alberto, and Thierry Verdier (2010): "The Economics of Cultural Transmission and Socialization." in the Handbook of Social Economics, Volume 1A, by Jess Benhabib, Alberto Bisin and Matthew O. Jackson.

[8] Blackwill, Robert D (2013): "Ideal Qualities of a Successful Diplomat." Paper, Belfer Center for Science and International Affairs, October 17.

[9] Bloch, Francis, and Harl Ryder (2000): "Two-Sided Search, Marriages, and Matchmakers." International Economic Review 41(1): 93-116.

[10] Borjas, George J. (1994): "The Economics of Immigration." Journal of Economic Literature 32(4): 1667-1717. 
[11] Borjas, George J. (1995): "The Economic Benefits from Immigration." The Journal of Economic Perspectives 9(2): 3-22.

[12] Borjas, George J. (2003): "The Labor Demand Curve is Downward Sloping: Reexamining the Impact of Immigration on the Labor Market." No. w9755. National Bureau of Economic Research.

[13] Boylan, Richard T. (1992): "Laws of Large Numbers for Dynamical Systems with Randomly Matched Individuals." Journal of Economic Theory 57(2): 473-504.

[14] Dewatripont, Mathias, and Jean Tirole (2005): "Modes of Communication." Journal of Political Economy 113(6): 1217-1238.

[15] Dustmann, Christian, Tommaso Frattini, and Ian Preston (2007): "A Study of Migrant Workers and the National Minimum Wage and Enforcement Issues that arise."

[16] Dustmann, Christian, Tommaso Frattini, and Ian P. Preston (2013): "The Effect of Immigration along the Distribution of Wages." The Review of Economic Studies 80(1): 145-173.

[17] Dustmann, Christian, and Tommaso Frattini (2014): "The Fiscal Effects of Immigration to the UK." The Economic Journal 124(580): 593-643.

[18] Ellison, Sara Fisher, and Wallace P. Mullin (2014): "Diversity, Social Goods Provision, and Performance in the Firm." Journal of Economics 83 Management Strategy 23(2): $465-481$.

[19] Giusta, Marina Della, Nigar Hashimzade, and Gareth D. Myles (2016): "Schooling and the Intergenerational Transmission of Values." Journal of Public Economic Theory.

[20] Hamilton, Barton H., Jack A. Nickerson, and Hideo Owan (2003): "Team Incentives and Worker Heterogeneity: An Empirical Analysis of the Impact of Teams on Productivity and Participation." Journal of political Economy 111(3): 465-497.

[21] Hansen, Lars Peter, and Thomas J. Sargent (2007): Robustness. Princeton university press. 
[22] Hardy, Godfrey H (1908): "Mendelian Proportions in a Mixed Population." Science: 49-50.

[23] Hong, Lu, and Scott E. Page (2004): "Groups of Diverse Problem Solvers can Outperform Groups of High-ability Problem Solvers." Proceedings of the National Academy of Sciences of the United States of America 101(46): 16385-16389.

[24] Hong, Lu, and Scott E. Page (2001): "Problem Solving by Heterogeneous Agents." Journal of Economic Theory 97(1): 123-163.

[25] Hoogendoorn, Sander, and Mirjam Van Praag (2012): "Ethnic Diversity and Team Performance: A Field Experiment.".

[26] Jackson, Matthew O., and Yiqing Xing (2014): "Culture-dependent Strategies in Coordination Games." Proceedings of the National Academy of Sciences 11(3): 10889-10896.

[27] Kemeny, Thomas (2012): "Cultural Diversity, Institutions, and Urban Economic Performance." Environment and Planning A 44(9): 2134-2152.

[28] Kemeny, Thomas (2014): "Immigrant Diversity and Economic Performance in Cities." International Regional Science Review: 0160017614541695.

[29] Ket, Willemien and Alvaro Sandroni (2015): "Challenging Conformity: A Case for Diversity?" CMS-EMS, Center for Mathematical Studies in Economics And Management Science, Discussion Paper \#1585, November 12.

[30] Lazear, Edward P. (1999a): "Globalisation and the Market for Team-mates." The Economic Journal 109(454): 15-40.

[31] Lazear, Edward P. (1999b): "Language and Culture." Journal of Political Economy 107(6): S95-S126.

[32] Li, Yiting (1998): "Middlemen and Private Information." Journal of Monetary Economics 42(1): 131-159.

[33] Niebuhr, Annekatrin (2010): "Migration and Innovation: Does Cultural Diversity Matter for Regional R\&D Activity?." Papers in Regional Science 89(3): 563-585. 
[34] O'Reilly, Charles A., Katherine Y. Williams, and Sigal Barsade (1997): "Demography and Group Performance: Does Diversity Help?" Graduate School of Business, Stanford University.

[35] Ottaviano, Gianmarco IP, and Giovanni Peri (2005): "Cities and Cultures." Journal of Urban Economics 58(2): 304-337.

[36] Ottaviano, Gianmarco IP, and Giovanni Peri (2006): "The Economic Value of Cultural Diversity: Evidence from US Cities." Journal of Economic Geography 6(1): 9-44.

[37] Page, Scott E. (2007): "The difference: How the Power of Diversity Creates Better Groups, Firms, Schools, and Societies."

[38] Petrongolo, Barbara, and Christopher A. Pissarides (2001): "Looking into the Black Box: A Survey of the Matching Function." Journal of Economic literature 39(2): 390431.

[39] Portes, Alejandro, and Rubn G. Rumbaut (2001): Legacies: The Story of the Immigrant Second Generation. Univ of California Press.

[40] Rubinstein, Ariel, and Asher Wolinsky (1987): "Middlemen." The Quarterly Journal of Economics: 581-594.

[41] Sauter, Disa A., Frank Eisner, Paul Ekman, and Sophie K. Scott (2010): "Cross-cultural Recognition of Basic Emotions through Nonverbal Emotional Vocalizations." Proceedings of the National Academy of Sciences 107(6): 2408-2412.

[42] Sparber, Chad (2009): "Racial Diversity and Aggregate Productivity in US industries: 1980-2000." Southern Economic Journal: 829-856.

[43] Stahl, Gnter K (2009): "Unraveling the Effects of Cultural Diversity in Teams: A Metaanalysis of Research on Multicultural Work Groups." Journal of International Business Studies 1: 20.

[44] Trax, Michaela, Stephan Brunow, and Jens Suedekum (2015): "Cultural Diversity and Plant-level Productivity." Regional Science and Urban Economics 53: 85-96. 
[45] Vasta, Ellie (2007): "From Ethnic Minorities to Ethnic Majority Policy: Multiculturalism and the Shift to Assimilationism in the Netherlands." Ethnic and Racial Studies 30(5): 713-740.

[46] Verkuyten, Maykel, and Jochem Thijs (2002): "Multiculturalism Among Minority and Majority Adolescents in the Netherlands." International Journal of Intercultural Relations 26(1): 91-108.

[47] Yava, Abdullah (1994): "Middlemen in Bilateral Search Markets." Journal of Labor Economics: 406-429. 